\title{
Entropy for Canonical Shifts. II
}

\author{
By \\ Marie CHODA* and Fumio Hial**†
}

\begin{abstract}
Let $N \subset M$ be a pair of factors. Associated with a conditional expectation $E$ from $M$ onto $N$ with finite index, we introduce the canonical shift $\Gamma$ on the von Neumann algebra $A$, with the canonical state $\phi$, generated by the tower of relative commutants for the basic constructions iterated from $E$. Related with the minimum index $[M: N]_{0}$, we investigate the entropy $h_{\phi}(\Gamma)$ of $\Gamma$ and the entropy $H_{\phi}(A \mid \Gamma(A))$ of $A$ relative to the subalgebra $\Gamma(A)$. The inequalities $h_{\phi}(\Gamma) \leq \log [M: N]_{0}$ and $\frac{1}{2} H_{\phi}(A \mid \Gamma(A)) \leq \log [M: N]_{0}$ hold in general. Furthermore when $E$ has the minimum index and $N \subset M$ has finite depth, we establish $h_{\phi}(\Gamma)=\frac{1}{2} H_{\phi}(A \mid \Gamma(A))=\log [M: N]_{0}$.
\end{abstract}

\section{Introduction}

Based on Connes' spatial theory [9] and Haagerup's theory on operator valued weights [15], Kosaki [24] extended Jones' index theory [22] for type $\mathrm{II}_{1}$ factors to that for conditional expectations between arbitrary factors. For a pair of factors $N \subset M$, let $\mathscr{E}(M, N)$ be the set of all faithful normal conditional expectations from $M$ onto $N$. Although Kosaki's index Index $E$ varies depending on $E \in \mathscr{E}(M, N)$, it was shown in [19] (also by Longo [30]) that if Index $E<\infty$ for some $E \in \mathscr{E}(M, N)$, then there exists a unique $E_{0} \in \mathscr{E}(M, N)$ which minimizes Index $E$ for $E \in \mathscr{E}(M, N)$. Then the minimum index $[M: N]_{0}$ for $N \subset M$ is defined as Index $E_{0}$.

Pimsner and Popa [33] extensively developed the entropy $H(M \mid N)$ for type $\mathrm{II}_{1}$ factors $N \subset M$ in connection with Jones' index $[M: N]$. Among other things, they showed the inequality $H(M \mid N) \leq \log [M: N]$ and obtained several characterizations for the equality. Also it was noted in [19] that $H(M \mid N)$ $=\log [M: N]$ is equivalent to $[M: N]=[M: N]_{0}$. The entropy $H(M \mid N)$ was first used in Connes and St $\phi$ rmer [12] to study the entropy of Kolmogorov-Sinai

Communicated by H. Araki, June 28, 1990.

1991 Mathematics Subject Classification: Primary 46L37, Secondly 46L40, 28D20.

* Department of Mathematics, Osaka Kyoiku University, Tennoji, Osaka 543, Japan.

** Division of Applied Mathematics, Research Institute of Applied Electricity, Hokkaido University, Sapporo 060, Japan.

Current address: Department of Mathematics, Ibaraki University, Mito, Ibaraki 310, Japan.

${ }^{\dagger}$ Research partially supported by Grant-in-Aid for Scientific Research. 
type for automorphisms of finite von Neumann algebras. Furthermore the notion of entropy for automorphisms was extended by Connes [10] and Connes, Narnhofer and Thirring [11] to the general setup of $C^{*}$-algebras or von Neumann algebras.

For von Neumann algebras $N \subset M$ and a faithful normal state $\varphi$ on $M$, the entropy $H_{\varphi}(M \mid N)$ is defined as in [10], which coincides with the above $H(M \mid N)$ when $\varphi$ is a trace. General properties of $H_{\varphi}(M \mid N)$ were given in [21]. When there exists $E \in \mathscr{E}(M, N)$ with $\varphi \circ E=\varphi$, another entropy $K_{\varphi}(M \mid N)$ was defined in [20] (also [23]) by taking account of Pimsner and Popa's estimate of $H(M \mid N)$. Given factors $N \subset M$ and $E \in \mathscr{E}(M, N)$, the relation between the entropy $K_{E}(M \mid N)$ and the minimum index $[M: N]_{0}$ was established in [20] in a way analogous to [33]. Here $K_{E}(M \mid N)=K_{\varphi}(M \mid N)$ independently of $\varphi$ with $\varphi \circ E=\varphi$, and $K_{E}(M \mid N)=H(M \mid N)$ when $N \subset M$ are type $\mathrm{II}_{1}$ factors and $E$ is the conditional expectation with respect to the trace.

The entropy $H(\sigma)$ for a $*$-endomorphism $\sigma$ of a finite von Neumann algebra $A$ was investigated in $[6,7]$ in connection with the entropy $H(A \mid \sigma(A))$ and the generalized index $\lambda(A, \sigma(A))$ introduced in [33]. Here the entropy for *endomorphisms can be defined in the same way as that for automorphims. For an inclusion $N \subset M$ of type $\mathrm{II}_{1}$ factors with finite index, Ocneanu [31] introduced a special kind of *-endormorphism $\Gamma$, called the canonical shift, on the tower of relative commutants induced by the tower of basic constructions. The canonical shift $\Gamma$ is extended on the von Neumann algebra $A$ generated by the tower of relative commutants, which becomes a typical example of 2-shifts. Under a certain assumption (equivalent to the equality $H(M \mid N)=\log [M: N])$, the following relations were obtained in [7]:

$$
H(A \mid \Gamma(A)) \leq 2 H(\Gamma) \leq \log \lambda(A, \Gamma(A))^{-1}=2 \log [M: N] .
$$

These numbers are all identical particularly when $N \subset M$ has finite depth. The aim of this paper is to extend the results in [7] to the canonical shift defined for a pair of arbitrary factors.

In $\S 1$ of this paper, for the reader's convenience, we list definitions and preliminaries on the index theory and the entropy theory. In particular, we note that the main results for automorphisms in $[10,11]$ remain valid also for $*$ endomorphisms. Now let $N \subset M$ be factors and $E \in \mathscr{E}(M, N)$ with Index $E$ $<\infty$. Then we obtain the basic construction for $E$ following [24], which consists of a factor $M_{1} \supset M$, a projection $e \in M_{1}$ with $M_{1}=\langle M, e\rangle$, and $E_{1} \in \mathscr{E}\left(M_{1}, M\right)$. In $\S 2$, we obtain an algebraic (up to isomorphisms) characterization of the basic construction $\left(M_{1}, e, E_{1}\right)$ for $E$. A similar characterization was given in [17].

Iterating the basic constructions from $E$, we obtain the tower of factors $N$ $\subset M=M_{0} \subset M_{1} \subset M_{2} \subset \cdots$ with projections $e_{n-1} \in M_{n}$ and $E_{n} \in \mathscr{E}\left(M_{n}, M_{n-1}\right)$ for $n \geq 1$. In $\S 3$, on the tower of relative commutants $M^{\prime} \cap M_{1} \subset M^{\prime} \cap M_{2} \subset \cdots$, 
we define the mirrorings $\gamma_{n}$ and the canonical shift $\Gamma$. To do so, we adopt a powerful idea of Longo's canonical endomorphism [28-30]. Here it is worth noting that the canonical shift $\Gamma$ on $\bigcup_{n}\left(M^{\prime} \cap M_{n}\right)$ is independent (up to isomorphisms) of the choice of $E \in \mathscr{E}(M, N)$. But taking the GNS representation associated with the state $\phi$ canonically determined by $\left\{E_{n}\right\}$, we extend $\Gamma$ (denoted by the same $\Gamma$ ) to a $*$-endomorphism of the von Neumann algebra $A$ generated by $\bigcup_{n}\left(M^{\prime} \cap M_{n}\right)$. We call $(A, \phi, \Gamma)$ the canonical shift associated with $E$. In particular, when $E=E_{0}$ (i.e. Index $E=[M: N]_{0}$ ), $\phi$ is a trace and Popa's analysis [36] on sequences of commuting squares can be applied to the tower of relative commutants in our setup. Thus $A$ is a type $\mathrm{II}_{1}$ factor when $E$ $=E_{0}$ and $N \subset M$ has finite depth.

In $\S \S 4$ and 5 , we establish relations among the entropy $h_{\phi}(\Gamma)$ of $\Gamma$, the entropy $H_{\phi}(A \mid \Gamma(A))$ relative to $\Gamma(A)$, and the minimum index $[M: N]_{0}$. The following inequalities hold in general:

$$
\begin{gathered}
h_{\phi}(\Gamma) \leq \frac{1}{2}\left\{K_{E}(M \mid N)+K_{E_{1}}\left(M_{1} \mid M\right)\right\} \leq \log [M: N]_{0}, \\
H_{\phi}(A \mid \Gamma(A)) \leq 2 \log [M: N]_{0} .
\end{gathered}
$$

We show that $E=E_{0} \quad$ if $h_{\phi}(\Gamma)=\log [M: N]_{0} \quad$ or if $H_{\phi}(A \mid \Gamma(A))$ $=2 \log [M: N]_{0}$. The inequality $H_{\phi}(A \mid \Gamma(A)) \leq 2 h_{\phi}(\Gamma)$ holds when $E$ $=E_{0}$. Furthermore when $E=E_{0}$ and $N \subset M$ has finite depth, we obtain

$$
H_{\phi}(A \mid \Gamma(A))=2 h_{\phi}(\Gamma)=\log \lambda_{\phi}(A, \Gamma(A))^{-1}=2 K_{E}(M \mid N)=2 \log [M: N]_{0} .
$$

Finally in $\S 6$, we give two typical examples to illustrate our main results.

\section{\$1. Preliminaries on Index and Entropy}

In this paper, all von Neumann algebras are assumed to be $\sigma$-finite. Let $M$ be a von Neumann algebra. The set of all faithful normal states on $M$ is denoted by $\mathscr{E}(M)$. Given a von Neumann subalgebra $N$ of $M$, we denote by $\mathscr{E}(M, N)$ the set of all faithful normal conditional expectations from $M$ onto $N$. In this section, we collect definitions and preliminaries on index and entropy for the reader's convenience.

\section{(1.1) Index for conditional expectations}

Let $N \subset M$ be von Neumann algebras on a Hilbert space $\mathscr{H}$. For each $E \in \mathscr{E}(M, N)$, there corresponds uniquely a faithful normal semifinite operator valued weight $E^{-1}$ from $N^{\prime}$ to $M^{\prime}$ by the equation $d(\varphi \circ E) / d \psi=d \varphi / d\left(\psi \circ E^{-1}\right)$ of spatial derivatives where $\varphi$ and $\psi$ are any faithful normal semifinite weights on $N$ and $M^{\prime}$, respectively (see [15], [37, 12.11]). When $N \subset M$ is a pair of factors, Kosaki [24] defined the index of $E$ by Index $E=E^{-1}(1)$. Kosaki's 
index extends Jones' index [22] in the sense that if $M$ is a finite factor and $E_{N}$ is the conditional expectation [39] onto a subfactor $N$ with respect to the trace, then Index $E_{N}$ coincides with Jones' index $[M: N]$.

The following formula (the best constant for Pimsner and Popa's inequality) serves as another definition of Index $E$. This formula for Jones' index is due to [33, Theorem 2.2], while for infinite factors it was obtained in several ways in [3, $16,27,30]$. The proof when Index $E<\infty$ is not difficult as shown in [27]. A nice proof of full generality is given in [26].

Theorem 1.1. Let $N \subset M$ be factors where $M$ is not finite dimensional. Then for every $E \in \mathscr{E}(M, N)$,

$$
(\operatorname{Index} E)^{-1}=\max \left\{\lambda \geq 0: E(x) \geq \lambda x, x \in M_{+}\right\} .
$$

Moreover (Index $E)^{-1}$ is always the best constant for the complete positivity of $E-\lambda \mathrm{id}_{M}$ including the case $M$ is finite dimensional.

\section{(1.2) Minimum index}

Given a pair of factors $N \subset M$, the value of Index $E$ depends on the choice of $E \in \mathscr{E}(M, N)$. But if Index $E<\infty$ for some $E \in \mathscr{E}(M, N)$, then the relative commutant $N^{\prime} \cap M$ is finite dimensional and Index $E<\infty$ for all $E \in \mathscr{E}(M, N)$ as noted in [19]. In this case, it was proved in [19] that there exists a unique $E_{0} \in \mathscr{E}(M, N)$ such that

$$
\text { Index } E_{0}=\min \{\operatorname{Index} E: E \in \mathscr{E}(M, N)\},
$$

which is characterized by the condition

$$
E_{0}^{-1}\left|N^{\prime} \cap M=\left(\operatorname{Index} E_{0}\right) E_{0}\right| N^{\prime} \cap M .
$$

In fact, $E_{0} \mid N^{\prime} \cap M$ becomes a trace on $N^{\prime} \cap M$. We define the minimum index $[M: N]_{0}$ for a pair $N \subset M$ by $[M: N]_{0}=\operatorname{Index} E_{0}$. Also let $[M: N]_{0}=\infty$ if $\mathscr{E}(M, N)=\varnothing$ or Index $E=\infty$ for all $E \in \mathscr{E}(M, N)$. Note [24, Theorem 4.4] that if Index $E<4$ for some $E \in \mathscr{E}(M, N)$, then $N^{\prime} \cap M=\mathrm{C}$ and hence $\mathscr{E}(M, N)$ $=\{E\}$. See $[18,20,30]$ for properties of the minimum index.

\section{(1.3) Commuting squares}

Consider a square

$$
\begin{aligned}
& N \subset M \\
& \cup \quad U \\
& B \subset A
\end{aligned}
$$

of von Neumann algebras and let $\varphi \in \mathscr{E}(M)$. Then the next proposition can be proved as $[14,4.2 .1]$.

Proposition 1.2. Assume that there exist conditional expectations $E: M \rightarrow N$, 
$F: M \rightarrow A$ and $G: M \rightarrow B$ with respect to $\varphi($ i.e. $\varphi \circ E=\varphi \circ F=\varphi \circ G=\varphi)$. Then the following conditions are equivalent:

(i) $E(A) \subset B$;

(ii) $E \circ F=G$;

(iii) $E \circ F=F \circ E$ and $A \cap N=B$;

(iv) $E|A=G| A$.

We say that

$$
\begin{aligned}
& N \subset M \\
& U \quad U \\
& B \subset A
\end{aligned}
$$

is a commuting square with respect to $\varphi$ if there exist the conditional expectations $E, F$ and $G$ as above and the equivalent conditions (i)-(iv) hold.

\section{(1.4) Entropies $H_{\varphi}(M \mid N)$ and $K_{\varphi}(M \mid N)$}

Let $N \subset M$ be von Neumann algebras with $\varphi \in \mathscr{E}(M)$. Following Connes [10], we define the entropy $H_{\varphi}(M \mid N)$ of $M$ relative to $N$ and $\varphi$ by

$$
H_{\varphi}(M \mid N)=\sup _{\left(\psi_{i}\right)} \sum_{i}\left\{S\left(\varphi, \psi_{i}\right)-S\left(\varphi\left|N, \psi_{i}\right| N\right)\right\}
$$

where the supremum is taken over all finite families $\left(\psi_{1}, \ldots, \psi_{n}\right)$ of $\psi_{i} \in M_{*}^{+}$with $\sum_{i} \psi_{i}=\varphi$. Here $S(\varphi, \psi)$ denotes the relative entropy of $\varphi, \psi \in M_{*}^{+}$, which was first introduced by Umegaki [40] in the semifinite case and extended by Araki $[1,2]$ to the general case. Particularly if $M$ is finite with a faithful normal trace $\tau$, $\tau(1)=1$, then $H(M \mid N)=H_{\tau}(M \mid N)$ is given by

$$
H(M \mid N)=\sup _{\left(x_{i}\right)} \sum_{i}\left\{\tau\left(\eta E_{N}\left(x_{i}\right)\right)-\tau\left(\eta x_{i}\right)\right\},
$$

where $\eta(t)=-t \log t$ on $[0, \infty), E_{N}: M \rightarrow N$ is the conditional expectation with respect to $\tau$, and the supremum is taken over all finite families $\left(x_{1}, \ldots, x_{n}\right)$ of $x_{i} \in M_{+}$with $\sum_{i} x_{i}=1$. See [21] for general properties of $H_{\varphi}(M \mid N)$.

When there exists $E \in \mathscr{E}(M, N)$ with $\varphi \circ E=\varphi$, another entropy $K_{\varphi}(M \mid N)$ of $M$ relative to $N$ and $\varphi$ was defined in [20] (also [23]) by

$$
K_{\varphi}(M \mid N)=-S(\hat{\omega}, \omega)
$$

where $\omega=\varphi \mid N^{\prime} \cap M$ and $\hat{\omega}=\varphi \circ\left(E^{-1} \mid N^{\prime} \cap M\right)$. Here unless $\hat{\omega}$ is bounded, the relative entropy $S(\hat{\omega}, \omega)$ is given by the infimum of $S\left(\omega^{\prime}, \omega\right)$ for $\omega^{\prime} \in\left(N^{\prime} \cap M\right)_{*}^{+}$ with $\omega^{\prime} \leq \hat{\omega}$. If $N$ is a factor, then $K_{\varphi}(M \mid N)$ is independent of the choice of $\varphi \in \mathscr{E}(M)$ with $\varphi \circ E=\varphi$, so that we write $K_{\varphi}(M \mid N)=K_{E}(M \mid N)$. Although the entropies $H_{\varphi}(M \mid N)$ and $K_{\varphi}(M \mid N)$ are not generally identical, we have $H(M \mid N)$ $=K_{\tau}(M \mid N)$ when $M$ is a type $\mathrm{II}_{1}$ factor. 


\section{(1.5) Relation between entropy and index}

Pimsner and Popa [33] established the relation between the entropy $H(M \mid N)$ and Jones' index $[M: N]$ for a pair of type $\mathrm{II}_{1}$ factors $N \subset M$. The entropy $K_{\varphi}(M \mid N)$ was investigated in [20] in connection with Kosaki's index and the minimum index for a pair of general factors. In the following, we state the main results in [20] restricting to the case of factors $N \subset M$. Here note that the centralizer $\left(N^{\prime} \cap M\right)_{E}$ of $E \in \mathscr{E}(M, N)$ is atomic whenever so is $N^{\prime} \cap M$. Also for each nonzero projection $q$ in $N^{\prime} \cap M, E(q)$ is a scalar and $E_{q} \in \mathscr{E}\left(M_{q}, N_{q}\right)$ is defined by $E_{q}(x)=E(q)^{-1} E(x) q, x \in M_{q}$.

Theorem 1.3. Let $E \in \mathscr{E}(M, N)$.

(1) If $N^{\prime} \cap M$ has a nonatomic part, then $K_{E}(M \mid N)=\infty$.

(2) If $N^{\prime} \cap M$ is atomic and $\left\{q_{i}\right\}$ is a set of atoms in $\left(N^{\prime} \cap M\right)_{E}$ with $\sum_{i} q_{i}=1$, then

$$
K_{E}(M \mid N)=\sum_{i} E\left(q_{i}\right) \log \frac{\operatorname{Index} E_{q_{i}}}{E\left(q_{i}\right)^{2}} .
$$

Theorem 1.4. (1) $K_{E}(M \mid N) \leq \log [M: N]_{0}$ for every $E \in \mathscr{E}(M, N)$.

(2) If $[M: N]_{0}<\infty$, then the following conditions for $E \in \mathscr{E}(M, N)$ are equivalent:

(i) Index $E=[M: N]_{0}$, i.e. $E=E_{0}$;

(ii) $K_{E}(M \mid N)=\log [M: N]_{0}$;

(iii) $K_{E}(M \mid N)=\log$ Index $E$;

(iv) Index $E_{q}=E(q)^{2}$ Index $E$ for every nonzero projection $q \in N^{\prime} \cap M$.

\section{(1.6) Entropy for *-endomorphisms}

After Connes and St $\phi$ rmer [12] developed the entropy of Kolmogorov-Sinai type for automorphisms of finite von Neumann algebras, Connes [10] and Connes, Narnhofer and Thirring [11] extended it to the general setup of $C^{*}$ algebras or von Neumann algebras. As in [6], to fix the notations, we briefly survey its definition extending to the case for $*$-endomorphisms of general von Neumann algebras.

Let $A$ be a von Neumann algebra with $\phi \in \mathscr{E}(A)$ and $\sigma$ a $*$-endomorphism of $A$ with $\phi \circ \sigma=\phi$. Then $\sigma$ is unital, injective and weakly continuous. For each $n \in \mathbb{N}$, we denote by $\mathscr{P}_{n}$ the set of all families $\Psi=\left(\psi_{i_{1}, \ldots, i_{n}}\right)_{i_{1}, \ldots, i_{n} \in \mathbb{N}}$ of $\psi_{i_{1}, \ldots, i_{n}} \in A_{*}^{+}$such that $\sum_{i_{1}, \ldots, i_{n}} \psi_{i_{1}, \ldots, i_{n}}=\phi$ and $\psi_{i_{1}, \ldots, i_{n}}=0$ except for a finite number of indices. For $\Psi \in \mathscr{P}_{n}, k \in\{1, \ldots, n\}$ and $j \in \mathbb{N}$, let

$$
\Psi_{j}^{k}=\sum_{i_{1}, \ldots, i_{k-1}, i_{k+1}, \ldots, i_{n}} \psi_{i_{1}, \ldots, i_{k-1}, j, i_{k+1}, \ldots, i_{n}} .
$$

Given finite dimensional subalgebras $B_{1}, \ldots, B_{n}$ of $A$, Connes [10] defined 


$$
H_{\phi}\left(B_{1}, \ldots, B_{n}\right)=\sup _{\Psi \in \mathscr{P}_{n}}\left\{\sum_{i_{1}, \ldots, i_{n}} \eta\left(\psi_{i_{1}, \ldots, i_{n}}(1)\right)+\sum_{k=1}^{n} \sum_{j} S\left(\phi\left|B_{k}, \Psi_{j}^{k}\right| B_{k}\right)\right\}
$$

Then the following is easy to check as in [6, Lemma 2]:

$$
H_{\phi}\left(\sigma\left(B_{1}\right), \ldots, \sigma\left(B_{n}\right)\right) \leq H_{\phi}\left(B_{1}, \ldots, B_{n}\right) .
$$

By this and [10, Théorème 5], the following limit exists for each finite dimensional subalgebra $B$ of $A$ :

$$
h_{\phi, \sigma}(B)=\lim _{n \rightarrow \infty} \frac{1}{n} H_{\phi}\left(B, \sigma(B), \ldots, \sigma^{n-1}(B)\right) .
$$

Now the entropy $h_{\phi}(\sigma)$ of $\sigma$ relative to $\phi$ is defined by the supremum of $h_{\phi, \sigma}(B)$ for all finite dimensional subalgebras $B$ of $A$.

The arguments in $[11, \S \mathrm{VII}]$ remain true also for $*$-endomorphism, so that we have:

Theorem 1.5. If $\left\{B_{k}\right\}$ is an increasing sequence of finite dimensional subalgebras with $A=\left(\bigcup_{k} B_{k}\right)^{\prime \prime}$ (so $A$ is approximately finite dimensional), then

$$
h_{\phi}(\sigma)=\lim _{k \rightarrow \infty} h_{\phi, \sigma}\left(B_{k}\right)
$$

Proposition 1.6. (1) $h_{\phi}(\sigma)=h_{\phi_{0} \theta}\left(\theta^{-1} \sigma \theta\right)$ for every automorphism $\theta$ of $A$.

(2) $h_{\phi}\left(\sigma^{n}\right) \leq n h_{\phi}(\sigma)$ for all $n \in \mathbf{N}$, and the equality holds if $A$ is approximately finite dimensional.

\section{§ 2. Basic Construction and Algebraic Basic Construction}

The concept of the basic construction invented by Jones is a core in the index theory $[22,24]$. In this section, we present some preliminary results on the (algebraic) basic construction, which will be useful in the next section.

First let us recall the procedure of the basic construction [24]. Let $N \subset M$ be factors and $E \in \mathscr{E}(M, N)$ with Index $E<\infty$. Choosing $\varphi \in \mathscr{E}(M)$ with $\varphi \circ E$ $=\varphi$, we represent $M$ standardly on $\mathscr{H}=\mathscr{H}_{\varphi}$ equipped with the natural cone $\mathscr{H}^{+}$and the associated modular conjugation $J$. Define the factor $M_{1}=J N^{\prime} J$ and the projection $e$ by $e(x \xi)=E(x) \xi, x \in M$, where $\varphi=\omega_{\xi}, \xi \in \mathscr{H}^{+}$. Then

$$
M_{1}=\langle M, e\rangle\left(=(M \cup\{e\})^{\prime \prime}\right) .
$$

Also $E_{1} \in \mathscr{E}\left(M_{1}, M\right)$ is defined by

$$
E_{1}(x)=(\operatorname{Index} E)^{-1} J E^{-1}(J x J) J, \quad x \in M_{1} .
$$

The construction of $\left(M_{1}, e, E_{1}\right)$ is called the basic construction for $E$. We write this procedure as follows: 


$$
N \underset{E}{\subset} M \underset{E_{1}}{\subset}{ }^{e} M_{1}
$$

The next proposition is a restatement of [25, Appendix I], which shows that the basic construction is canonical up to spatial isomorphisms.

Proposition 2.1. Let $\hat{N} \subset \hat{M}$ be factors with $\hat{E} \in \mathscr{E}(\hat{M}, \hat{N})$. Let $\theta: M \rightarrow \hat{M}$ be an isomorphism such that $\theta(N)=\hat{N}$ and $\theta \circ E=\hat{E} \circ \theta$ (hence Index $\hat{E}=$ Index $E$ $<\infty)$. Let

$$
\hat{N} \underset{\overleftarrow{E}}{\subset} \hat{M} \underset{\overleftarrow{E}_{1}}{\subset} \hat{e}_{1}^{\hat{M}}
$$

be the basic construction for $\hat{E}$ defined on a Hibert space $\hat{\mathscr{H}}$ with the conjugation $\hat{J}$. Then there exists a unitary $u: \mathscr{H} \rightarrow \hat{\mathscr{H}}$ such that

(i) $u x u^{*}=\theta(x), x \in M$,

(ii) $u J u^{*}=\hat{J}$,

(iii) $u e u^{*}=\hat{e}$,

(iv) $u M_{1} u^{*}=\hat{M}_{1}$,

(v) $\operatorname{Ad}(u) \circ E_{1}=\hat{E}_{1} \circ \operatorname{Ad}(u)$ on $M_{1}$.

Now let us introduce the notion of algebraic basic constructions. Let $N$ $\subset M \subset M_{1}$ be factors, $e \in M_{1}$ a projection, $E \in \mathscr{E}(M, N)$ and $E_{1} \in \mathscr{E}\left(M_{1}, M\right)$. We call $\left(M_{1}, e, E_{1}\right)$ an algebraic basic construction for $E$ if

(1) $M_{1}=\langle M, e\rangle$,

(2) $E_{1}(e)=\lambda 1(\lambda>0)$

(3) exe $=E(x) e, x \in M$.

In this case, we have Index $E<\infty$ by Theorem 1.1 because for $x \in M_{+}$

$$
\lambda E(x)=E_{1}(E(x) e)=E_{1}(e x e) \geq E_{1}\left(x^{1 / 2} e\right)^{*} E_{1}\left(x^{1 / 2} e\right)=\lambda^{2} x .
$$

Note [24, Lemma 3.2 and (8)] that the basic construction for $E$ is an algebraic one. The next proposition shows the uniqueness of algebraic basic constructions for $E$ up to isomorphisms. In other words, the above (1)-(3) algebraically characterize the basic construction for $E$ (see [34, Proposition 1.2] for more elegant characterizations in the type $\mathrm{II}_{1}$ case). A similar result was obtained by Hamachi and Kosaki [17, Theorem 8].

Proposition 2.2. Besides $\left(M_{1}, e, E_{1}\right)$ for $E \in \mathscr{E}(M, N)$ as above, let $\tilde{N} \subset \tilde{M}$ $\subset \tilde{M}_{1}$ be factors, $\tilde{e} \in \tilde{M}_{1}$ a projection, $\tilde{E} \in \mathscr{E}(\tilde{M}, \tilde{N})$ and $\tilde{E}_{1} \in \mathscr{E}\left(\tilde{M}_{1}, \tilde{M}\right)$ such that $\left(\tilde{M}_{1}, \tilde{e}, \tilde{E}_{1}\right)$ is an algebraic basic construction for $\tilde{E}$. If $\theta: M \rightarrow \tilde{M}$ is an isomorphism such that $\theta(N)=\tilde{N}$ and $\theta \circ E=\tilde{E} \circ \theta$, then there exists a unique isomorphism $\theta_{1}: M_{1} \rightarrow \tilde{M}_{1}$ such that

(i) $\theta_{1}(x)=\theta(x), x \in M$,

(ii) $\theta_{1}(e)=\tilde{e}$,

(iii) $\theta_{1} \circ E_{1}=\tilde{E}_{1} \circ \theta_{1}$. 
Proof. Since Index $E<\infty$ as remarked above, we can take the basic construction for $E$. So we may assume that $\left(M_{1}, e, E_{1}\right)$ is the basic construction (in the spatial sense) for $E$. Let $\widetilde{E}_{1}(\tilde{e})=\tilde{\lambda} 1(\tilde{\lambda}>0)$, while $E_{1}(e)$ $=\lambda 1$ with $\lambda=(\operatorname{Index} E)^{-1}$. Let $\varphi \in \mathscr{E}(M)$ and define $\psi \in \mathscr{E}\left(M_{1}\right), \tilde{\psi} \in \mathscr{E}\left(\tilde{M}_{1}\right)$ by $\psi$ $=\varphi \circ E_{1}, \tilde{\psi}=\varphi \circ \theta^{-1} \circ \widetilde{E}_{1}$. According to [33, Proposition 1.3] and [41, 2.5.3], there exists a basis $\left\{m_{1}, \ldots, m_{n}\right\}$ in $M$ for $E$ which satisfies $\sum_{i} m_{i} e m_{i}^{*}=1$. The existence of such a basis shows that $M_{1}$ is the linear span of $\{a e b: a, b \in M\}$ (see the proof of [33, Proposition 1.5]). For any $a_{i}, b_{i} \in M, 1 \leq i \leq k$, we have

$$
\psi\left(\sum_{i} a_{i} e b_{i}\right)=\lambda \varphi\left(\sum_{i} a_{i} b_{i}\right)=\mu \tilde{\psi}\left(\sum_{i} \theta\left(a_{i}\right) \tilde{e} \theta\left(b_{i}\right)\right)
$$

where $\mu=\lambda \tilde{\lambda}^{-1}$. Therefore a map $\theta_{1}: M_{1} \rightarrow \tilde{M}_{1}$ is well defined by

$$
\theta_{1}\left(\sum_{i} a_{i} e b_{i}\right)=\sum_{i} \theta\left(a_{i}\right) \tilde{e} \theta\left(b_{i}\right), \quad a_{i}, b_{i} \in M .
$$

Then (ii) holds and $\theta_{1}$ is a $*$-homomorphism. Since $\psi=\mu \tilde{\psi} \circ \theta_{1}, \theta_{1}$ is normal and hence injective. We get

$$
\theta_{1}(1) \tilde{e}=\theta_{1}(e)=\tilde{e} \theta_{1}(1)
$$

and since $\theta_{1}(1)=\sum_{i} \theta\left(m_{i}\right) \tilde{e} \theta\left(m_{i}^{*}\right)$,

$$
\theta_{1}(1) \theta(x)=\theta_{1}(x)=\theta(x) \theta_{1}(1), \quad x \in M,
$$

so that $\theta_{1}(1)=1$, showing (i). Moreover the linear span of $\{\tilde{a} \tilde{e} \tilde{b}: \tilde{a}, \tilde{b} \in \tilde{M}\}$ is the $*$-algebra generated by $\tilde{M} \cup\{\tilde{e}\}$, so that $\theta_{1}$ is surjective. Since

$$
\left(\theta_{1} \circ E_{1}\right)(a e b)=\lambda \theta(a b)=\mu\left(\tilde{E}_{1} \circ \theta_{1}\right)(a e b), \quad a, b \in M,
$$

we get $\mu=1$ and (iii) holds. It is immediate that (i) and (ii) uniquely determine $\theta_{1}$.

Proposition 2.2 shows that algebraic properties of the basic construction automatically become those of an algebraic basic construction $\left(M_{1}, e, E_{1}\right)$ for $E$. So we have for instance

(4) Index $E=\operatorname{Index} E_{1}=\lambda^{-1}$ for $\lambda$ in (2),

(5) $N=M \cap\{e\}^{\prime}$,

(6) $e \in\left(N^{\prime} \cap M_{1}\right)_{E^{\circ} E_{1}}$, the centralizer of $E \circ E_{1}$.

Furthermore we have:

Proposition 2.3. In any representation of $N \subset M \subset M_{1}$,

(7) $E^{-1}(e)=1$,

(8) $e x e=\lambda E_{1}^{-1}(x) e, x \in M^{\prime}$.

Proof. Since (7) and (8) hold for the basic construction, it suffices by 
Proposition 2.2 to show that the validity of (7) and (8) is preserved under isomorphisms, that is, if $\alpha: M_{1} \rightarrow \tilde{M}_{1}$ is an isomorphism and if $\tilde{M}=\alpha(M), \tilde{N}$ $=\alpha(N), \tilde{E}=\alpha_{0} \circ E \circ \alpha_{0}^{-1}\left(\alpha_{0}=\alpha \mid M\right), \widetilde{E}_{1}=\alpha \circ E_{1} \circ \alpha^{-1}$ and $\tilde{e}=\alpha(e)$, then (7) and (8) imply

(7') $\tilde{E}^{-1}(\tilde{e})=1$,

$\left(8^{\prime}\right) \quad \tilde{e} x \tilde{e}=\lambda \tilde{E}_{1}^{-1}(x) \tilde{e}, x \in \tilde{M}^{\prime}$.

We may separately consider an amplification, an induction and a spatial isomorphism. The first two cases are easily shown by [20, Propositions 1.7 and 1.5]. The final case is obvious.

From now on, let $N \subset M$ be always a pair of factors with $[M: N]_{0}$ $<\infty$. By iterating the basic construction from $E \in \mathscr{E}(M, N)$, we obtain the tower

$$
N \underset{E}{\subsetneq} M=M_{0} \underset{E_{1}}{\subset} M_{1} \underset{E_{2}}{\subset} M_{2} \subset \cdots
$$

of factors $M_{n}$ together with conditional expectations $E_{n} \in \mathscr{E}\left(M_{n}, M_{n-1}\right)$. Then we have the commuting square property as follows.

Proposition 2.4. Let $\varphi_{0} \in \mathscr{E}(M)$ with $\varphi_{0}{ }^{\circ} E=\varphi_{0}$, and define $\varphi_{n} \in \mathscr{E}\left(M_{n}\right)$ by

$$
\varphi_{n}=\varphi_{0} \circ E_{1} \circ \cdots \circ E_{n}, \quad n \geq 1 .
$$

Then for every $0 \leq j \leq k \leq n$

$$
\begin{array}{ccc}
M_{k} & \subset & M_{n} \\
\bigcup & & \bigcup \\
M_{j}^{\prime} \cap M_{k} & \subset M_{j}^{\prime} \cap M_{n}
\end{array}
$$

is a commuting square with respect to $\varphi_{n}$.

Proof. Let $E_{k, n}=E_{k+1} \circ \cdots \circ E_{n}$ which is the conditional expectation $M_{n}$ $\rightarrow M_{k}$ with respect to $\varphi_{n}$. Hence by [38], we have

$$
\sigma_{t}^{\varphi_{n}}\left(M_{j}^{\prime} \cap M_{n}\right)=M_{j}^{\prime} \cap M_{n}, \quad \sigma_{t}^{\varphi_{n}}\left(M_{j}^{\prime} \cap M_{k}\right)=M_{j}^{\prime} \cap M_{k}, \quad t \in \mathbb{R},
$$

so that there exist the conditional expectations $M_{n} \rightarrow M_{j}^{\prime} \cap M_{n}$ and $M_{n} \rightarrow M_{j}^{\prime} \cap M_{k}$ with respect to $\varphi_{n}$. Now it is easy to check that $E_{k, n}\left(M_{j}^{\prime} \cap M_{n}\right)=M_{j}^{\prime} \cap M_{k}$, implying the conclusion by Proposition 1.2.

Let $E_{0} \in \mathscr{E}(M, N)$ be such that Index $E_{0}=[M: N]_{0}$. Then the characterization of $E_{0}$ in (1.2) immediately shows the following:

Proposition 2.5. $E=E_{0}$ if and only if $E(x)=E_{1}\left(J x^{*} J\right)$ for all $x \in N^{\prime} \cap M$.

The next important result on the minimum index for the tower is proved by Kosaki and Longo [26]. 
Theorem 2.6. Suppose $E=E_{0}$. Then for every $0 \leq k<n$, $E_{k+1} \circ \cdots \circ E_{n} \in \mathscr{E}\left(M_{n}, M_{k}\right)$ gives the minimum index for $M_{k} \subset M_{n}$; equivalently $\left[M_{n}: M_{k}\right]_{0}=[M: N]_{0}^{n-k}$. In particular, $E_{k+1} \circ \cdots \circ E_{n} \mid M_{k}^{\prime} \cap M_{n}$ is a trace.

\section{§3. Definitions of Mirrorings and Canonical Shift}

Ocneanu [31] introduced the important concepts of the mirrorings and the canonical shift on the tower of relative commutants for the inclusion of type $\mathrm{II}_{1}$ factors with finite index. The aim of this section is to present precise definitions of the mirrorings and the canonical shift which are available to the inclusion $N$ $\subset M$ of general factors.

Given $E \in \mathscr{E}(M, N)$ where Index $E<\infty$ by assumption, let

$$
N \underset{E}{\subsetneq} M=M_{0} \underset{E_{1}}{\stackrel{e_{0}}{\subset}} M_{1} \underset{E_{2}}{\check{c}^{e_{1}}} M_{2} \underset{E_{3}}{\subset^{e_{2}}} M_{3} \subset \cdots
$$

be the tower of basic constructions iterated from $E$. Here for each $n \geq 1, M_{n}$ is standardly represented on a Hilbert space $\mathscr{H}_{n}$ with the modular conjugation $J_{n}$, so that

$$
\begin{aligned}
& M_{n+1}=J_{n} M_{n-1}^{\prime} J_{n}=\left\langle M_{n}, e_{n}\right\rangle, \\
& E_{n+1}(x)=\lambda J_{n} E_{n}^{-1}\left(J_{n} x J_{n}\right) J_{n}, \quad x \in M_{n+1},
\end{aligned}
$$

where $\lambda=(\operatorname{Index} E)^{-1}$. Then we obtain the tower

$$
M^{\prime} \cap M_{1} \subset M^{\prime} \cap M_{2} \subset M^{\prime} \cap M_{3} \subset \cdots
$$

of relative commutant algebras, which is an increasing sequence of finite dimensional algebras.

For each fixed $n \geq 1$, let us define

$$
\begin{array}{ll}
\tilde{M}_{k}=J_{n} M_{2 n-k}^{\prime} J_{n}, & n+1 \leq k \leq 2 n, \\
\tilde{e}_{k}=J_{n} e_{2 n-k} J_{n}, & n+1 \leq k \leq 2 n-1, \\
\tilde{E}_{k}(x)=\lambda J_{n} E_{2 n-k+1}^{-1}\left(J_{n} x J_{n}\right) J_{n}, & x \in \tilde{M}_{k}, n+2 \leq k \leq 2 n,
\end{array}
$$

where $M_{2 n-k}^{\prime}$ and $E_{2 n-k+1}^{-1}$ are defined for $M_{2 n-k} \subset M_{2 n-k+1}$ represented on $\mathscr{H}_{n}$. Then

$$
\begin{aligned}
& M_{n} \subset M_{n+1}=\tilde{M}_{n+1} \subset \tilde{M}_{n+2} \subset \cdots \subset \tilde{M}_{2 n}, \\
& \tilde{e}_{k-1} \in \tilde{M}_{k}, \tilde{E}_{k} \in \mathscr{E}\left(\tilde{M}_{k}, \tilde{M}_{k-1}\right), \quad n+2 \leq k \leq 2 n,
\end{aligned}
$$

and we have:

Lemma 3.1. For every $n+1 \leq k \leq 2 n-1,\left(\tilde{M}_{k+1}, \tilde{e}_{k}, \tilde{E}_{k+1}\right)$ is an algebraic basic construction for $\tilde{E}_{k}$ where $\tilde{E}_{n+1}=E_{n+1}$. 
Proof. Let us check three conditions for the algebraic basic construction. Since $M_{2 n-k-1}=M_{2 n-k} \cap\left\{e_{2 n-k}\right\}^{\prime}, \quad \tilde{M}_{k+1}=\left\langle\tilde{M}_{k}, \tilde{e}_{k}\right\rangle$. Since $E_{2 n-k}^{-1}\left(e_{2 n-k}\right)=1$ by Proposition 2.3 (7),

$$
\tilde{E}_{k+1}\left(\tilde{e}_{k}\right)=\lambda J_{n} E_{2 n-k}^{-1}\left(e_{2 n-k}\right) J_{n}=\lambda 1 \text {. }
$$

Since by Proposition 2.3 (8)

$$
e_{2 n-k} x e_{2 n-k}=\lambda E_{2 n-k+1}^{-1}(x) e_{2 n-k}, \quad x \in M_{2 n-k}^{\prime},
$$

we get for $x \in \tilde{M}_{k}$

$$
\tilde{e}_{k} x \tilde{e}_{k}=\lambda J_{n} E_{2 n-k+1}^{-1}\left(J_{n} x J_{n}\right) e_{2 n-k} J_{n}=\tilde{E}_{k}(x) \tilde{e}_{k} .
$$

Lemma 3.1 enables us to apply Proposition 2.2 recursively, so that we have the following:

Proposition 3.2. For every $n \geq 1$, there exists a unique isomorphism $\theta_{n}: M_{2 n}$ $\rightarrow \tilde{M}_{2 n}$ such that

(1) $\theta_{n}(x)=x, x \in M_{n+1}$,

(2) $\theta_{n}\left(M_{k}\right)=\tilde{M}_{k}, n+2 \leq k \leq 2 n$,

(3) $\theta_{n}\left(e_{k}\right)=\tilde{e}_{k}, n+1 \leq k \leq 2 n-1$,

(4) $\theta_{n} \circ E_{k}=\widetilde{E}_{k} \circ \theta_{n}$ on $M_{k}, n+2 \leq k \leq 2 n$.

Using $\theta_{n}$ in Proposition 3.2, we now define antiautomorphisms $\gamma_{n}$ of $M^{\prime} \cap M_{2 n}, n \geq 1$, by

$$
\gamma_{n}(x)=\theta_{n}^{-1}\left(J_{n} \theta_{n}\left(x^{*}\right) J_{n}\right), \quad x \in M^{\prime} \cap M_{2 n} .
$$

(Also let $\gamma_{0}=\mathrm{id}$ on $M^{\prime} \cap M=$ C.) We obviously have $\gamma_{n} \circ \gamma_{n}=\mathrm{id}_{M^{\prime} \cap M_{2 n}}$. These $\gamma_{n}$ are called the mirrorings on the tower of relative commutants, which extend those in [31]. The next proposition shows that the sequence $\left\{\gamma_{n}\right\}$ of mirrorings is determined (up to isomorphisms) independently of the choice of the tower.

Proposition 3.3. Besides the tower and $\left\{\gamma_{n}\right\}$ described above, let

$$
N \underset{E}{\subsetneq} M=\hat{M}_{0} \underset{\hat{E}_{1}}{\subset} \hat{e}_{0}^{\hat{e}_{0}} \hat{M}_{1} \underset{\hat{E}_{2}}{\subsetneq \hat{e}_{1}} \hat{M}_{2} \subset \cdots
$$

be another tower of basic constructions from $E$, and $\left\{\hat{\gamma}_{n}\right\}$ be the associated sequence of mirrorings. Then there exists an isomorphism $\Theta: \bigcup_{n} M_{n} \rightarrow \bigcup_{n} \hat{M}_{n}$ such that for every $n \geq 1$

(1) $\Theta(x)=x, x \in M$,

(2) $\Theta\left(M_{n}\right)=\hat{M}_{n}$,

(3) $\Theta \circ E_{n}=\hat{E}_{n} \circ \Theta$ on $M_{n}$,

(4) $\Theta \circ \gamma_{n}=\hat{\gamma}_{n} \circ \Theta$ on $M^{\prime} \cap M_{2 n}$.

Proof. Let $\hat{M}_{n}$ be standardly represented on a Hilbert space $\hat{\mathscr{H}}_{n}$ with the 
conjugation $\hat{J}_{n}$. Applying Proposition 2.1 recursively, we have a sequence $\left\{u_{n}: n \geq 0\right\}$ of unitaries $u_{n}: \mathscr{H}_{n} \rightarrow \hat{\mathscr{H}}_{n}$ such that for every $n \geq 0$

(i) $u_{n} x u_{n}^{*}=u_{n-1} x u_{n-1}^{*}, x \in M_{n}$ (with convention $u_{-1}=1$ ),

(ii) $u_{n} J_{n} u_{n}^{*}=\hat{J}_{n}$,

(iii) $u_{n} e_{n} u_{n}^{*}=\hat{e}_{n}$,

(iv) $u_{n} M_{n+1} u_{n}^{*}=\hat{M}_{n+1}$,

(v) $\operatorname{Ad}\left(u_{n}\right) \circ E_{n+1}=\hat{E}_{n+1} \circ \operatorname{Ad}\left(u_{n}\right)$ on $M_{n+1}$.

Thus an isomorphism $\Theta: \bigcup_{n} M_{n} \rightarrow \bigcup_{n} \hat{M}_{n}$ can be defined by $\Theta(x)=u_{n} x u_{n}^{*}$, $x \in M_{n}, n \geq 0$. Then (1)-(3) are immediate. Let us prove (4). Besides $\theta_{n}: M_{2 n}$ $\rightarrow \tilde{M}_{2 n}\left(=J_{n} M^{\prime} J_{n}\right)$, let $\hat{\theta}_{n}: \hat{M}_{2 n} \rightarrow \hat{J}_{n} M^{\prime} \hat{J}_{n}, n \geq 1$, be isomorphisms as in Proposition 3.2 associated with the second tower. For each $n \geq 1$, we can define the isomorphism $\Theta_{2 n}: M_{2 n} \rightarrow \hat{M}_{2 n}$ by $\Theta_{2 n}=\hat{\theta}_{n}^{-1} \circ \operatorname{Ad}\left(u_{n}\right) \circ \theta_{n}$ because $u_{n}\left(J_{n} M^{\prime} J_{n}\right) u_{n}^{*}$ $=\hat{J}_{n} M^{\prime} \hat{J}_{n}$. Then by Proposition 3.2 together with (i)-(v), we have the following:

(1 $\left.{ }^{\circ}\right) \Theta_{2 n}=\operatorname{Ad}\left(u_{n}\right)$ on $M_{n+1}$,

(2。 $\Theta_{2 n}\left(M_{k}\right)=\hat{M}_{k}, n+2 \leq k \leq 2 n$,

(3) $\Theta_{2 n}\left(e_{k}\right)=\hat{e}_{k}, n+1 \leq k \leq 2 n-1$,

(4) $\Theta_{2 n} \circ E_{k}=\hat{E}_{k} \circ \Theta_{2 n}, n+2 \leq k \leq 2 n$.

Since the above $\left(1^{\circ}\right)-\left(4^{\circ}\right)$ are the conditions which uniquely determine the isomorphism $\Theta \mid M_{2 n}$, it follows that $\Theta_{2 n}=\Theta \mid M_{2 n}$. Therefore we get for $x \in M^{\prime} \cap M_{2 n}$

$$
\left(\Theta \circ \gamma_{n}\right)(x)=\hat{\theta}_{n}^{-1}\left(u_{n} J_{n} \theta_{n}\left(x^{*}\right) J_{n} u_{n}^{*}\right)=\hat{\theta}_{n}^{-1}\left(\hat{J}_{n} \hat{\theta}_{n}\left(\Theta(x)^{*}\right) \hat{J}_{n}\right)=\left(\hat{\gamma}_{n} \circ \Theta\right)(x),
$$

as desired.

In the proof of the next proposition, we adopt a key idea of Longo's canonical endomorphism investigated in [28-30].

Proposition 3.4. For every $n \geq 1, \gamma_{n+1} \circ \gamma_{n}=\gamma_{n} \circ \gamma_{n-1}$ on $M^{\prime} \cap M_{2 n-2}$.

Proof. In view of Proposition 3.3, we may show in the particular choice of the tower. First assume that $M$ is infinite (hence so is $N$ ). Since the assumption of finite index implies that $N^{\prime}$ is $\sigma$-finite on the standard Hilbert space $\mathscr{H}$ for $M$, we can choose $\xi_{0} \in \mathscr{H}$ which is cyclic and separating for both $M$ and $N$ (see [13]). Let $J_{M}$ and $J_{N}$ be the modular conjugations associated with $\xi_{0}$ for $M$ and $N$, respectively, and let $W=J_{M} J_{N}$. We define the basic construction $\left(M_{1}, e_{0}, E_{1}\right)$ for $E$ via the natural cone associated with $\xi_{0}$ for M. Since

$$
M_{1}=J_{M} N^{\prime} J_{M}=W N W^{*},
$$

$M_{1}$ is standard on $\mathscr{H}$ with the conjugation $J_{1}=W J_{N} W^{*}$ associated with $\xi_{0}$. Hence the basic construction for $E_{1}$ can be defined via the natural cone associated with $\xi_{0}$ for $M_{1}$, so that we have two steps of the tower 


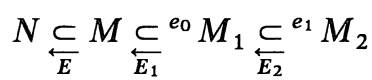

on the same $\mathscr{H}$. Here

$$
M_{2}=W J_{N} W^{*} M^{\prime} W J_{N} W^{*}=W M W^{*} .
$$

Now define for $n \geq 1$

$$
\begin{aligned}
& M_{2 n}=W^{n} M W^{* n}, M_{2 n+1}=W^{n+1} N W^{* n+1}\left(=W^{n} M_{1} W^{* n}\right), \\
& J_{2 n}=W^{n} J_{M} W^{* n}, J_{2 n+1}=W^{n+1} J_{N} W^{* n+1}\left(=W^{n} J_{1} W^{* n}\right), \\
& e_{2 n}=W^{n} e_{0} W^{* n}, e_{2 n+1}=W^{n} e_{1} W^{* n}, \\
& E_{2 n}=W^{n} E\left(W^{* n} \cdot W^{n}\right) W^{* n}, E_{2 n+1}=W^{n} E_{1}\left(W^{* n} \cdot W^{n}\right) W^{* n} .
\end{aligned}
$$

Then it is easy to see that the tower

$$
N \underset{E}{\subsetneq} M \underset{E_{1}}{\subset}{ }^{e_{0}} M_{1} \underset{E_{2}}{\subset^{e_{1}}} M_{2} \underset{E_{3}}{\subset^{e_{2}}} M_{3} \subset \cdots
$$

is a realization of the tower of basic constructions from $E$. For this tower, the isomorphisms $\theta_{n}: M_{2 n} \rightarrow \tilde{M}_{2 n}$ in Proposition 3.2 are simply $\theta_{n}=\operatorname{id}_{M_{2 n}}\left(\tilde{M}_{2 n}\right.$ $\left.=M_{2 n}\right)$. Therefore

$$
\gamma_{n}(x)=J_{n} x^{*} J_{n}, \quad x \in M^{\prime} \cap M_{2 n}, \quad n \geq 1 .
$$

This shows that for $n \geq 1$

$$
\gamma_{n}\left(\gamma_{n-1}(x)\right)=W x W^{*}, \quad x \in M^{\prime} \cap M_{2 n-2},
$$

and particularly $\gamma_{n+1} \circ \gamma_{n}=\gamma_{n} \circ \gamma_{n-1}$ on $M^{\prime} \cap M_{2 n-2}$.

Next assume that $M$ is finite. Taking the tensor product of the tower from $E$ with any infinite factor $P$, we obtain

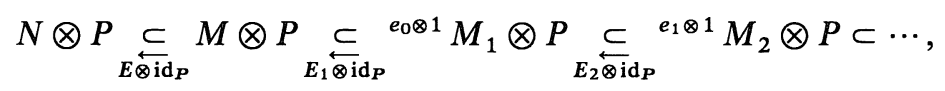

which is really the tower of basic constructions from $E \otimes \mathrm{id}_{P}$. Since the isomorphisms in Proposition 3.2 for the tensored tower are $\theta_{n} \otimes \mathrm{id}_{P}: M_{2 n} \otimes P$ $\rightarrow \tilde{M}_{2 n} \otimes P$ and $M^{\prime} \cap M_{2 n}=(M \otimes P)^{\prime} \cap\left(M_{2 n} \otimes P\right)$, the mirrorings for the tensored tower coincide with those for the original tower. Thus the desired equality follows from the infinite case.

The next proposition is a partial extension of [34, Theorem 2.6] (also [31]).

Proposition 3.5. For every $0 \leq k<n, E_{n+1} \circ \cdots \circ E_{2 n-k}$ is the basic construction of $E_{k+1} \circ \cdots \circ E_{n}$; more precisely 


$$
\left(E_{n+1} \circ \cdots \circ E_{2 n-k}\right)(x)=\lambda^{n-k} J_{n}\left(E_{k+1} \circ \cdots \circ E_{n}\right)^{-1}\left(J_{n} \theta_{n}(x) J_{n}\right) J_{n}
$$

for all $x \in M_{2 n-k}$ with $\theta_{n}$ in Proposition 3.2.

Proof. By the proof of Proposition 3.3, it suffices to show in the particular choice of the tower. When $M$ is infinite, we take the tower specified in the proof of Proposition 3.4 where $\theta_{n}=\mathrm{id}_{M_{2 n}}$. The case $k=n-1$ is just the definition of the basic construction. Suppose the equality holds for some $0<k$ $<n$. Then for every $x \in M_{2 n-k+1}$, we have

$$
\begin{aligned}
\left(E_{n+1} \circ \cdots \circ E_{2 n-k+1}\right)(x) & =\lambda^{n-k+1} J_{n}\left(E_{k+1} \circ \cdots \circ E_{n}\right)^{-1}\left(E_{k}^{-1}\left(J_{n} x J_{n}\right)\right) J_{n} \\
& =\lambda^{n-k+1} J_{n}\left(E_{k} \circ \cdots \circ E_{n}\right)^{-1}\left(J_{n} x J_{n}\right) J_{n},
\end{aligned}
$$

because

$$
E_{2 n-k+1}(x)=\lambda J_{n} E_{k}^{-1}\left(J_{n} x J_{n}\right) J_{n}
$$

as easily checked. Hence the conclusion follows by induction. When $M$ is finite, we can do as in the proof of Proposition 3.4.

Given the tower from $E$, Proposition 3.4 enables us to define a *endomorphism $\Gamma$ of $\bigcup_{n}\left(M^{\prime} \cap M_{n}\right)$ by

$$
\Gamma(x)=\gamma_{n+1}\left(\gamma_{n}(x)\right), \quad x \in M^{\prime} \cap M_{2 n},
$$

which is called the canonical shift on the tower of relative commutants. In view of the proof of Proposition 3.4, we know that the canonical shift $\Gamma$ as well as the mirrorings $\gamma_{n}$ can be constructed apart from the choice of $E \in \mathscr{E}(M, N)$. In this sense, $\Gamma$ is canonical for the inclusion $N \subset M$ rather than for $E$. Now the faithful state $\phi$ on $\bigcup_{n}\left(M^{\prime} \cap M_{n}\right)$ is defined by

$$
\phi\left|M^{\prime} \cap M_{n}=E_{1} \circ \ldots \circ E_{n}\right| M^{\prime} \cap M_{n}, \quad n \geq 1 .
$$

Then we have:

Proposition 3.6. (1) $\gamma_{n}\left(M_{j}^{\prime} \cap M_{k}\right)=M_{2 n-k}^{\prime} \cap M_{2 n-j}, 0 \leq j \leq k \leq 2 n$.

(2) $\Gamma\left(M_{k}^{\prime} \cap M_{n}\right)=M_{k+2}^{\prime} \cap M_{n+2}, 0 \leq k \leq n$.

(3) $\Gamma^{k} \circ \gamma_{n}=\gamma_{n+k}$ on $M^{\prime} \cap M_{2 n}, k, n \geq 0$.

(4) $\phi \circ \Gamma=\phi$ on $\bigcup_{n}\left(M^{\prime} \cap M_{n}\right)$.

(5) If $E=E_{0}$, then $\phi \circ \gamma_{n}=\phi$ on $M^{\prime} \cap M_{2 n}, n \geq 0$.

Proof. The case of $M$ being finite follows from the infinite case by taking the tensor product with an infinite factor. So let $M$ be infinite. It suffices as before to show for the tower specified in the proof of Proposition 3.4. Then (1)(3) are directly checked for $\Gamma(x)=W x W^{*}$ and $\gamma_{n}(x)=J_{n} x^{*} J_{n}, x \in M^{\prime} \cap M_{2 n}$. (In fact, $W^{k} J_{n}=J_{n} W^{* k}=J_{n+k}$.)

(4) Proposition 3.5 implies that 


$$
\left(E_{k+1} \circ \cdots \circ E_{n}\right)(x)=\lambda^{n-k} J_{n}\left(E_{n+1} \circ \cdots \circ E_{2 n-k}\right)^{-1}\left(J_{n} x J_{n}\right) J_{n}
$$

for all $x \in M_{n}, n>k \geq 0$. Hence for every $x \in M^{\prime} \cap M_{n}$, we get

$$
\begin{aligned}
\phi(x) & =\left(E_{1} \circ \cdots \circ E_{n+1}\right)(x) \\
& =\lambda^{n+1} J_{n+1}\left(E_{n+2} \circ \cdots \circ E_{2 n+2}\right)^{-1}\left(J_{n+1} x J_{n+1}\right) J_{n+1} .
\end{aligned}
$$

Since $J_{n+1} \times J_{n+1} \in M_{n+2}^{\prime} \cap M_{2 n+2}$, we get $E_{n+2}^{-1}\left(J_{n+1} \times J_{n+1}\right)=\lambda^{-1} J_{n+1} x J_{n+1}$. Therefore

$$
\begin{aligned}
\phi(x) & =\lambda^{n} J_{n+1}\left(E_{n+3} \circ \cdots \circ E_{2 n+2}\right)^{-1}\left(J_{n+1} x J_{n+1}\right) J_{n+1} \\
& =J_{n+1} J_{n+2}\left(E_{3} \circ \cdots \circ E_{n+2}\right)\left(J_{n+2} J_{n+1} x J_{n+1} J_{n+2}\right) J_{n+2} J_{n+1} \\
& =\left(E_{3} \circ \cdots \circ E_{n+2}\right)\left(W x W^{*}\right)=\phi(\Gamma(x)) .
\end{aligned}
$$

(5) Let $E=E_{0}$ and $x \in M^{\prime} \cap M_{2 n}$. Combining Proposition 2.5, Theorem 2.6 and Proposition 3.5, we have

$$
\begin{aligned}
\phi(x) & =\left(E_{1} \circ \cdots \circ E_{2 n}\right)(x)=\left(E_{2 n+1} \circ \cdots \circ E_{4 n}\right)\left(J_{2 n} x^{*} J_{2 n}\right) \\
& =\phi\left(\gamma_{2 n}(x)\right)=\phi\left(\Gamma^{n}\left(\gamma_{n}(x)\right)\right)=\phi\left(\gamma_{n}(x)\right)
\end{aligned}
$$

by (3) and (4).

Let us extend $\Gamma$ to a *-endomorphism of the von Neumann algebra generated by $\bigcup_{n}\left(M^{\prime} \cap M_{n}\right)$. So define

$$
A=\pi_{\phi}\left(\bigcup_{n}\left(M^{\prime} \cap M_{n}\right)\right)^{\prime \prime}
$$

where $\pi_{\phi}$ is the GNS representation of $\bigcup_{n}\left(M^{\prime} \cap M_{n}\right)$ associated with $\phi$. Further let $\bar{\phi}$ be the normal extension of $\phi$ on $A$, so that $\bar{\phi}\left(\pi_{\phi}(x)\right)=\phi(x)$, $x \in \bigcup_{n}\left(M^{\prime} \cap M_{n}\right)$.

The inclusion $N \subset M$ is said to have finite depth if

$$
\sup _{n} \operatorname{dim} Z\left(M^{\prime} \cap M_{n}\right)<\infty
$$

where $Z\left(M^{\prime} \cap M_{n}\right)$ denotes the center of $M^{\prime} \cap M_{n}$ (this condition does not depend on the choice of $E$ ).

Proposition 3.7. (1) $\bar{\phi}$ is a faithful normal state on $A$.

(2) There exists a unique *-endomorphism $\bar{\Gamma}$ of $A$ such that $\bar{\phi} \circ \bar{\Gamma}=\bar{\phi}$ and $\bar{\Gamma}\left(\pi_{\phi}(x)\right)=\pi_{\phi}(\Gamma(x)), x \in \bigcup_{n}\left(M^{\prime} \cap M_{n}\right)$.

(3) If $E=E_{0}$, then $\bar{\phi}$ is a faithful normal trace on $A$.

(4) If $E=E_{0}$ and $N \subset M$ has finite depth, then $A$ is a type $\mathrm{I}_{1}$ factor.

Proof. (1) Let $A^{0}$ be the $C^{*}$-completion of $\bigcup_{n}\left(M^{\prime} \cap M_{n}\right)$ with the extension $\phi^{0}$ of $\phi$. Then $\pi_{\phi}$ is nothing but the GNS representation of $A^{0}$ associated with 
$\phi^{0}$. Letting $\phi_{n}=\phi \mid M^{\prime} \cap M_{n}$, since $\sigma_{t}^{\phi_{n+1}} \mid M^{\prime} \cap M_{n}=\sigma_{t}^{\phi_{n}}$ for all $n \geq 1$, we obtain a one-parameter automorphism group $\sigma_{t}^{0}$ of $A^{0}$ such that $\sigma_{t}^{0} \mid M^{\prime} \cap M_{n}=\sigma_{t}^{\phi_{n}}$, $n \geq 1$. Hence it follows (see $[4,5.3 .9]$ for instance) that the normal extension $\bar{\phi}$ of $\phi^{0}$ is faithful.

(2) follows from Proposition 3.6 (4).

(3) follows from Theorem 2.6.

(4) Suppose $E=E_{0}$. Then Popa's arguments in [36, §2] work in our setup as well. In fact, the results [36, Proposition 2.1, Corollaries 2.2 and 2.3] (also $[14,4.6 .3])$ hold for $\lambda=[M: N]_{0}^{-1}$ and $\left\{B_{n}=M^{\prime} \cap M_{n}: n \geq 0\right\}$, when we consider the dimension vector and the trace vector of $B_{n}$ with respect to the trace $\phi$ together with the inclusion matrix of $B_{n} \subset B_{n+1}$. Thus the same proof as [36, Corollary 2.5] implies the desired conclusion under the finite depth assumption.

Since $\pi_{\phi}$ faithfully imbeds $\bigcup_{n}\left(M^{\prime} \cap M_{n}\right)$ in $A$, we consider $\bigcup_{n}\left(M^{\prime} \cap M_{n}\right)$ as a subalgebra of $A$ and denote $\bar{\Gamma}, \bar{\phi}$ by $\Gamma, \phi$ again. We call the $*$-endomorphism $\Gamma$ extended on $A$, or more precisely $(A, \phi, \Gamma)$, the canonical shift associated with $E$. In particular, let $N \subset M$ be type $\mathrm{II}_{1}$ factors and $(A, \phi, \Gamma)$ the canonical shift associated with the conditional expectation $E_{N}: M \rightarrow N$ with respect to the trace. Then $\phi$ is a trace whether $E_{N}=E_{0}$ or not. This $\Gamma$ is the canonical shift for $N \subset M$ investigated in [7].

On the lines of [29, Theorem 5.1], we have the ergodic property of $\Gamma$ extending [7, Proposition 21].

Proposition 3.8. $\bigcap_{k=1}^{\infty} \Gamma^{k}(A)=\mathbf{C}$.

Proof. Let $\|\cdot\|_{\phi}$ be the norm on $A$ induced by $\phi$, i.e. $\|x\|_{\phi}$ $=\phi\left(x^{*} x\right)^{1 / 2}$. Let $x \in \bigcap_{k} \Gamma^{k}(A)$. For any $\varepsilon>0$, there exist $k$ and $y \in M^{\prime} \cap M_{2 k}$ such that $\|x-y\|_{\phi}<\varepsilon$. For every $n \geq 2 k$, Proposition 2.4 shows that

$$
\begin{array}{ccc}
M^{\prime} \cap M_{2 k} & \subset & M^{\prime} \cap M_{n} \\
U & & U \\
\mathbf{C} & \subset M_{2 k}^{\prime} \cap M_{n}
\end{array}
$$

is a commuting square with respect to $\phi \mid M^{\prime} \cap M_{n}$. By Proposition 3.6 (2), $\Gamma^{k}(A)$ is generated by $\bigcup_{n}\left(M_{2 k}^{\prime} \cap M_{n}\right)$. Hence we see that

$$
\begin{array}{ccc}
M^{\prime} \cap M_{2 k} & \subset & A \\
U & & \bigcup \\
\mathbf{C} & \subset \Gamma^{k}(A)
\end{array}
$$

is a commuting square with respect to $\phi$. So there exists the conditional expectation $F: A \rightarrow \Gamma^{k}(A)$ with respect to $\phi$, which satisfies $F\left(M^{\prime} \cap M_{2 k}\right)$ $=\mathbf{C}$. Since $F(x)=x$ and $F(y)=\phi(y)$, we get 


$$
\|x-\phi(x)\|_{\phi} \leq\|F(x-y)\|_{\phi}+|\phi(y-x)| \leq 2\|x-y\|_{\phi}<2 \varepsilon,
$$

which implies $x \in \mathbb{C}$.

\section{\$4. Entropy $\boldsymbol{h}_{\phi}(\Gamma)$}

Let $(A, \phi, \Gamma)$ be the canonical shift associated with $E \in \mathscr{E}(M, N)$ defined in the previous section. Let $B_{n}=M^{\prime} \cap M_{n}$ and $\phi_{n}=\phi \mid B_{n}$ for $n \geq 0$. Then $\left\{B_{n}\right\}$ is an increasing sequence of finite dimensional subalgebras of $A$ with $A$ $=\left(\bigcup_{n} B_{n}\right)^{\prime \prime}$. The aim of this section is to establish the relation between the entropy $h_{\phi}(\Gamma)$ and the minimum index $[M: N]_{0}$.

Lemma 4.1. (1) For every $n, m \geq 0,\left(\bigcup_{j=0}^{m} \Gamma^{j}\left(B_{n}\right)\right)^{\prime \prime}$ is included in $B_{n+2 m}$.

(2) Let $k_{n}=\left[\frac{n+1}{2}\right]$. Then for every $n, m \geq 0, \Gamma^{(m+1) k_{n}}\left(B_{n}\right)$ commutes with $\left(\bigcup_{j=0}^{m k_{n}} \Gamma^{j}\left(B_{n}\right)\right)^{\prime \prime}$ and $\phi(x y)=\phi(x) \phi(y)$ for all $x \in\left(\bigcup_{j=0}^{m k_{n}} \Gamma^{j}\left(B_{n}\right)\right)^{\prime \prime}$ and $y \in \Gamma^{(m+1) k_{n}}\left(B_{n}\right)$.

(3) The conditional expectation $A \rightarrow \Gamma^{j}\left(B_{n}\right)$ with respect to $\phi$ exists for every $n, j \geq 0$, and

$$
\begin{array}{cccc}
B_{n} & \subset & A \\
U & & U \\
\Gamma\left(B_{n-2}\right) & \subset \Gamma\left(B_{n}\right)
\end{array}
$$

is a commuting square with respect to $\phi$ for every $n \geq 2$.

(4) $\Gamma\left(B_{2 n}\right)=\gamma_{n+1}\left(B_{2 n}\right)$ for all $n \geq 0$.

Moreover if $E=E_{0}$, then $\Gamma$ is a 2-shift on the tower $\left\{B_{n}\right\}$ in the sense of [7].

Proof. (1) and (2) follow from Proposition 3.6 (2).

(3) By the proof of Proposition 3.7 (1) and [4, 5.3.4], the conditional expectation $A \rightarrow B_{n}$ with respect to $\phi$ exists for every $n \geq 0$. Then Proposition 2.4 shows the desired conclusions (see the proof of Proposition 3.8).

(4) is obvious from Proposition 3.6 (3).

By Propositions 3.6 (5) and 3.7 (3), the above (1)-(4) show the last statement.

Proposition 4.2. $h_{\phi}(\Gamma)=\lim _{n \rightarrow \infty} \frac{1}{n} H_{\phi}\left(B_{2 n}\right)=\lim _{n \rightarrow \infty} \frac{1}{n} S\left(\phi_{2 n}\right)$ where $S\left(\phi_{n}\right)$ is the entropy of $\phi_{n}$.

Proof. For each $n, m \geq 1$, let $B=\left(\bigcup_{j=0}^{m-1} \Gamma^{j k_{n}}\left(B_{n}\right)\right)^{\prime \prime}$ where $k_{n}=\left[\frac{n+1}{2}\right]$, and $\left\{q_{i}: 1 \leq i \leq l\right\}$ be a set of atoms in the centralizer of $\phi_{n}$ with $\sum_{i} q_{i}$ $=1$. Furthermore let $q_{i}^{j}=\Gamma^{(j-1) k_{n}}\left(q_{i}\right)$ for $1 \leq i \leq l$ and $1 \leq j \leq m$. Then by Proposition 3.6 (4) and Lemma 4.1 (2), $\left\{q_{i_{1}}^{1} \cdots q_{i_{m}}^{m}: 1 \leq i_{1}, \ldots, i_{m} \leq l\right\}$ is a set of atoms in the centralizer of $\phi \mid B$ such that $\sum_{i_{1}, \ldots, i_{m}} q_{i_{1}}^{1} \cdots q_{i_{m}}^{m}=1$ and $\phi\left(q_{i_{1}}^{1} \cdots q_{i_{m}}^{m}\right)$ 
$=\phi\left(q_{i_{1}}^{1}\right) \cdots \phi\left(q_{i_{m}}^{m}\right)$. Hence by Lemma 4.1 and [10, Théorème 5(E)] (also [11, Corollary VIII.8]), we get

$$
\begin{aligned}
H_{\phi}\left(B_{n}, \Gamma^{k_{n}}\left(B_{n}\right), \ldots, \Gamma^{(m-1) k_{n}}\left(B_{n}\right)\right) & =S(\phi \mid B)=\sum_{i_{1}, \ldots, i_{m}} \eta\left(\phi\left(q_{i_{1}}^{1} \cdots q_{i_{m}}^{m}\right)\right) \\
& =m S\left(\phi_{n}\right)=m H_{\phi}\left(B_{n}\right) .
\end{aligned}
$$

Now the proof is the same as [7, Theorem 1] in view of Theorem 1.5 and Proposition 1.6 (2).

Theorem 4.3. (1) $h_{\phi}(\Gamma) \leq \frac{1}{2}\left\{K_{E}(M \mid N)+K_{E_{1}}\left(M_{1} \mid M\right)\right\} \leq \log [M: N]_{0}$.

(2) If $h_{\phi}(\Gamma)=\log [M: N]_{0}$, then $E=E_{0}$.

(3) Suppose $N \subset M$ has finite depth. Then the following conditions are equivalent:

(i) $E=E_{0}$;

(ii) $h_{\phi}(\Gamma)=\log [M: N]_{0}$;

(iii) $h_{\phi}(\Gamma)=\log$ Index $E$.

Proof. (1) For each $n \geq 1$, choose a set $\left\{q_{i}^{(2 n)}\right\}$ of atoms in the centralizer of $\phi_{2 n}$ with $\sum_{i} q_{i}^{(2 n)}=1$. Since the centralizer of $E_{1} \circ \cdots \circ E_{2 n}$ is nothing but that of $\phi_{2 n}$ and

$$
\operatorname{Index}\left(E_{1} \circ \cdots \circ E_{2 n}\right)_{q_{i}^{(2 n)}} \geq 1,
$$

Theorem 1.3 (2) implies that

$$
K_{E_{1} \cdots \cdots E_{2 n}}\left(M_{2 n} \mid M\right) \geq 2 \sum_{i} \eta\left(\phi_{2 n}\left(q_{i}^{(2 n)}\right)\right)=2 S\left(\phi_{2 n}\right) .
$$

Furthermore by [20, Theorem 5.1 (1)] and [21, Proposition 8.1], we have

$$
K_{E_{1} \ldots \ldots E_{2 n}}\left(M_{2 n} \mid M\right) \leq \sum_{j=1}^{2 n} K_{E_{j}}\left(M_{j} \mid M_{j-1}\right)=n\left\{K_{E}(M \mid N)+K_{E_{1}}\left(M_{1} \mid M\right)\right\}
$$

Thus Proposition 4.2 implies the first inequality. Also we get the second inequality by Theorem 1.4 (1).

(2) Suppose $h_{\phi}(\Gamma)=\log [M: N]_{0}$. Then $K_{E}(M \mid N)=\log [M: N]_{0}$ holds, which is equivalent to $E=E_{0}$ by Theorem 1.4 (2).

(3) In view of (2), it suffices to show that (i) implies (ii) under the finite depth condition. So suppose $N \subset M$ has finite depth and $E=E_{0}$. Then there exists $n_{0}$ such that $\operatorname{dim} Z\left(B_{2 n_{0}+2}\right)=\operatorname{dim} Z\left(B_{2 n_{0}}\right)$. Since [36, Corollary 2.3] holds for $\left\{B_{n}\right\}$ in our setup as noted in the proof of Proposition 3.7 (4), the trace vector of $B_{2 n}$ with respect to the trace $\phi$ is given by $\left(\lambda^{n-n_{0}} s_{k}\right)_{k}$ for any $n \geq n_{0}$, where $\lambda$ $=[M: N]_{0}^{-1}$ and $\left(s_{k}\right)$ is the trace vector of $B_{2 n_{0}}$ with respect to $\phi$. Let $s$ $=\max _{k} s_{k}$ and $n \geq n_{0}$. Since $\phi_{2 n}\left(q_{i}^{(2 n)}\right) \leq \lambda^{n-n_{0}} s$, we have by Theorems 2.6 and 
$1.4(2)$

$$
\begin{aligned}
\operatorname{Index}\left(E_{1} \circ \cdots \circ E_{2 n}\right)_{q_{i}^{(2 n)}} & =\phi_{2 n}\left(q_{i}^{(2 n)}\right)^{2} \operatorname{Index}\left(E_{1} \circ \ldots \circ E_{2 n}\right) \\
& \leq\left(\lambda^{n-n_{0}} s\right)^{2} \lambda^{-2 n}=\left(\lambda^{-n_{0}} s\right)^{2}
\end{aligned}
$$

for all $i$. Therefore by Theorems 2.6, 1.4 (2) and 1.3 (2), we have

$$
\begin{aligned}
2 n \log [M: N]_{0} & =\log \left[M_{2 n}: M\right]_{0}=K_{E_{1} \ldots \circ E_{2 n}}\left(M_{2 n} \mid M\right) \\
& \leq \sum_{i} \phi_{2 n}\left(q_{i}^{(2 n)}\right) \log \frac{\left(\lambda^{-n_{0}} s\right)^{2}}{\phi_{2 n}\left(q_{i}^{(2 n)}\right)^{2}}=2 S\left(\phi_{2 n}\right)+2 \log \left(\lambda^{-n_{0}} s\right),
\end{aligned}
$$

so that $h_{\phi}(\Gamma) \geq \log [M: N]_{0}$ by Proposition 4.2.

Specializing Theorem 4.3 to the type $\mathrm{II}_{1}$ case, we have:

Corollary 4.4. Let $N \subset M$ be type $\mathrm{II}_{1}$ factors and $H(\Gamma)$ the entropy of the canonical shift $\Gamma$ for $N \subset M$. Then:

(1) $H(\Gamma) \leq \frac{1}{2}\left\{H(M \mid N)+H\left(M_{1} \mid M\right)\right\} \leq \log [M: N]_{0} \leq \log [M: N]$.

(2) If $H(\Gamma)=\log [M: N]_{0}$, then $[M: N]=[M: N]_{0}$ and $E_{M^{\prime} \cap M_{1}}\left(e_{0}\right)$ $=[M: N]^{-1} 1$ where $E_{M^{\prime} \cap M_{1}}$ is the conditional expectation $M_{1} \rightarrow M^{\prime} \cap M_{1}$ with respect to the trace.

(3) If $N \subset M$ has finite depth, then

$$
H(\Gamma)=H(M \mid N)=\log [M: N]_{0}=\log [M: N] .
$$

Proof. Let $E_{N}$ be the conditional expectation $M \rightarrow N$ with respect to the trace. We know by [33, Corollary 4.5] and [19] that $E_{N}=E_{0}$ (i.e. $[M: N]$ $\left.=[M: N]_{0}\right)$ if and only if $E_{M^{\prime} \cap M_{1}}\left(e_{0}\right)=[M: N]^{-1} 1$. According to $[36$, Corollary 3.7], if $N \subset M$ has finite depth, then $E_{M^{\prime} \cap M_{1}}\left(e_{0}\right)=[M: N]^{-1} 1$ automatically holds. Thus the corollary is the specialization of Theorem 4.3.

\section{§5. Entropy $H_{\phi}(A \mid \Gamma(A))$}

Let $(A, \phi, \Gamma)$ be the canonical shift associated with $E \in \mathscr{E}(M, N)$. Let $B_{n}$ and $\phi_{n}$ be as in $\S 4$, and $C_{n}=M_{2}^{\prime} \cap M_{n}\left(=\Gamma\left(B_{n-2}\right)\right), n \geq 2$. In this section, we investigate the entropy $H_{\phi}(A \mid \Gamma(A))$ in connection with $[M: N]_{0}$ and $h_{\phi}(\Gamma)$.

The entropy $H_{\phi}\left(B_{n} \mid C_{n}\right)$ is given in [10] by

$$
H_{\phi}\left(B_{n} \mid C_{n}\right)=\sup _{\left(\psi_{k}\right)} \sum_{k}\left\{S\left(\phi\left|B_{n}, \psi_{k}\right| B_{n}\right)-S\left(\phi\left|C_{n}, \psi_{k}\right| C_{n}\right)\right\}
$$

where the supremum is taken over all finite families $\left(\psi_{k}\right)$ of $\psi_{k} \in A_{*}^{+}$with $\sum_{k} \psi_{k}$ $=\phi$. But we have $H_{\phi}\left(B_{n} \mid C_{n}\right)=H_{\phi_{n}}\left(B_{n} \mid C_{n}\right)$ by Lemma 4.1 (3). Proposition 2.4 and [21, Proposition $2.12(1)]$ show the following: 
Proposition 5.1. $H_{\phi}(A \mid \Gamma(A))=\lim _{n \rightarrow \infty} H_{\phi}\left(B_{n} \mid C_{n}\right)$ increasingly.

Proposition 5.2. $h_{\phi}(\Gamma) \leq H_{\phi}(A \mid \Gamma(A))$.

Proof. By [21, Proposition 2.2 (1)] and Proposition 3.6, we get for $n \geq 1$

$$
H_{\phi}\left(B_{2 n}\right) \leq H_{\phi}\left(B_{2 n} \mid C_{2 n}\right)+H_{\phi}\left(C_{2 n}\right)=H_{\phi}\left(B_{2 n} \mid C_{2 n}\right)+H_{\phi}\left(B_{2 n-2}\right) .
$$

This implies that

$$
\frac{1}{n} H_{\phi}\left(B_{2 n}\right) \leq \frac{1}{n} \sum_{j=1}^{n} H_{\phi}\left(B_{2 j} \mid C_{2 j}\right)
$$

Hence the desired inequality follows from Propositions 4.2 and 5.1.

Theorem 5.3. (1) $H_{\phi}(A \mid \Gamma(A)) \leq 2 \log [M: N]_{0}$.

(2) If $H_{\phi}(A \mid \Gamma(A))=2 \log [M: N]_{0}$, then $E=E_{0}$.

(3) Suppose $N \subset M$ has finite depth. Then $E=E_{0}$ if and only if $H_{\phi}(A \mid \Gamma(A))=2 \log [M: N]_{0}$.

Proof. (1) Let

$$
N \underset{E_{0}}{\subset} \underset{E_{0,1}}{\subset}{ }^{e_{0}} M_{1} \underset{E_{0,2}}{\subset^{e_{1}}} M_{2} \subset \cdots
$$

be the tower of basic constructions iterated from $E_{0}$. Here we can assume as remarked before Proposition 3.6 that the factors $M_{n}$ are the same as those in the tower iterated from $E$. For $n \geq 1$, let $\tau_{n}=E_{0,1} \circ \ldots \circ E_{0, n} \mid B_{n}$ which is a trace by Theorem 2.6, and let $h_{n}=d\left(E_{n} \mid M_{n-1}^{\prime} \cap M_{n}\right) / d\left(E_{0, n} \mid M_{n-1}^{\prime} \cap M_{n}\right)$. Since $E_{n}$ $=h_{n}^{1 / 2} E_{0, n} h_{n}^{1 / 2}\left(=E_{0, n}\left(h_{n}^{1 / 2} \cdot h_{n}^{1 / 2}\right)\right)$ by $[8$, Théorème 5.3], we get

$$
E_{1} \circ \cdots \circ E_{n}=\left(h_{1} \cdots h_{n}\right)^{1 / 2}\left(E_{0,1} \circ \cdots \circ E_{0, n}\right)\left(h_{1} \cdots h_{n}\right)^{1 / 2},
$$

so that $d \phi_{n} / d \tau_{n}=h_{1} \cdots h_{n}$. For each fixed $n \geq 2$, we denote by $F$ and $F_{0}$ the conditional expectations $B_{n} \rightarrow C_{n}$ with respect to $\phi_{n}$ and $\tau_{n}$, respectively. For $1 \leq k \leq n$, let us define $E_{0, k}^{\prime} \in \mathscr{E}\left(M_{k-1}^{\prime}, M_{k}^{\prime}\right)$ by $E_{0, k}^{\prime}=\lambda E_{0, k}^{-1}$ where $\lambda=[M: N]_{0}^{-1}$ and $E_{0, k}^{-1}$ is defined on the standard Hilbert space for $M_{n}$. Then it follows that

$$
M_{n}^{\prime} \underset{E_{0, n}^{\prime}}{\subset} M_{n-1}^{\prime} \underset{E_{0, n-1}^{\prime}}{\subset} e_{n-1}^{e_{n-2}} M_{n-2}^{\prime} \subset \cdots \subset \subset^{e_{2}} M_{1}^{\prime} \underset{E_{0,1}^{\prime}}{\subset}{ }^{e_{1}} M^{\prime}
$$

is $n$ steps of algebraic basic constructions. Since by Theorem 2.6

$$
E_{0, n}^{\prime} \circ \cdots \circ E_{0,1}^{\prime}\left|B_{n}=\lambda^{n}\left(E_{0,1} \circ \cdots \circ E_{0, n}\right)^{-1}\right| B_{n}=\tau_{n},
$$

we have $F_{0}=E_{0,2}^{\prime} \circ E_{0,1}^{\prime} \mid B_{n}$, so that

$$
\frac{d\left(\phi_{n} \mid C_{n}\right)}{d\left(\tau_{n} \mid C_{n}\right)}=F_{0}\left(h_{1} \cdots h_{n}\right)=h_{3} \cdots h_{n}
$$


Hence the cocycle derivative $\left[D F: D F_{0}\right]_{t}$ of $F$ and $F_{0}$ is computed as follows (see $[8,15])$ :

$$
\begin{aligned}
{\left[D F: D F_{0}\right]_{t} } & =\left[D\left(\tau_{n} \circ F\right): D\left(\tau_{n} \circ F_{0}\right)\right]_{t} \\
& =\left[D\left(\phi_{n} \circ F\right): D\left(\tau_{n} \circ F\right)\right]_{t}^{*}\left[D\left(\phi_{n} \circ F\right): D\left(\tau_{n} \circ F_{0}\right)\right]_{t} \\
& =\left[D\left(\phi_{n} \mid C_{n}\right): D\left(\tau_{n} \mid C_{n}\right)\right]_{t}^{*}\left[D \phi_{n}: D \tau_{n}\right]_{t} \\
& =\left(h_{3} \cdots h_{n}\right)^{-i t}\left(h_{1} \cdots h_{n}\right)^{i t}=\left(h_{1} h_{2}\right)^{i t} .
\end{aligned}
$$

Now let $\psi_{1}, \ldots, \psi_{m} \in\left(B_{n}\right)_{*}^{+}$be faithful with $\sum_{k} \psi_{k}=\phi_{n}$. Then

$$
\begin{aligned}
{\left[D\left(\psi_{k} \circ F\right): D \psi_{k}\right]_{t} } & =\left[D\left(\psi_{k} \circ F\right): D\left(\psi_{k} \circ F_{0}\right)\right]_{t}\left[D\left(\psi_{k} \circ F_{0}\right): D \psi_{k}\right]_{t} \\
& =\left[D F: D F_{0}\right]_{t}\left[D\left(\psi_{k} \circ F_{0}\right): D \psi_{k}\right]_{t} \\
& =\left(h_{1} h_{2}\right)^{i t}\left[D\left(\psi_{k} \circ F_{0}\right): D \psi_{k}\right]_{t} .
\end{aligned}
$$

Hence by [32, Theorem 4], we get

$$
\begin{aligned}
S\left(\psi_{k} \circ F, \psi_{k}\right) & =i \lim _{t \rightarrow+0} \frac{1}{t} \psi_{k}\left(\left[D\left(\psi_{k} \circ F\right): D \psi_{k}\right]_{t}-1\right) \\
& =S\left(\psi_{k} \circ F_{0}, \psi_{k}\right)+\psi_{k}\left(\log h_{1} h_{2}\right) .
\end{aligned}
$$

Since by Theorem 1.1

$$
F_{0}(x) \geq\left(\operatorname{Index}\left(E_{0,2}^{\prime} \circ E_{0,1}^{\prime}\right)\right)^{-1} x=\lambda^{2} x, \quad x \in\left(B_{n}\right)_{+},
$$

we have $\psi_{k} \circ F_{0} \geq \lambda^{2} \psi_{k}$, so that

$$
\sum_{k} S\left(\psi_{k}^{\circ} F_{0}, \psi_{k}\right) \leq \sum_{k} \psi_{k}(1) \log \lambda^{-2}=2 \log [M: N]_{0}
$$

Therefore

$$
\sum_{k} S\left(\psi_{k}^{\circ} F, \psi_{k}\right) \leq 2 \log [M: N]_{0}+\tau_{2}\left(\eta\left(h_{1} h_{2}\right)\right)
$$

By the lower semicontinuity of the relative entropy ([2, Theorem 3.7]), the above inequality holds for any $\psi_{1}, \ldots, \psi_{m} \in\left(B_{n}\right)_{*}^{+}$with $\sum_{k} \psi_{k}=\phi_{n}$. This implies by [21, Lemma 2.6$]$ that

$$
H_{\phi}\left(B_{n} \mid C_{n}\right) \leq 2 \log [M: N]_{0}+\tau_{2}\left(\eta\left(h_{1} h_{2}\right)\right) .
$$

Since $\tau_{2}\left(\eta\left(h_{1} h_{2}\right)\right) \leq \eta\left(\tau_{2}\left(h_{1} h_{2}\right)\right)=0$, the desired inequality follows from Proposition 5.1.

(2) Suppose $H_{\phi}(A \mid \Gamma(A))=2 \log [M: N]_{0}$. Then $\tau_{2}\left(\eta\left(h_{1} h_{2}\right)\right)=0$ and hence $h_{1} h_{2}=1$ by the strict concavity of $\eta$. This implies $E_{1} \circ E_{2}=E_{0,1} \circ E_{0,2}$, so that $E_{1}=E_{0,1}$, equivalently $E=E_{0}$.

(3) Suppose $N \subset M$ has finite depth and $E=E_{0}$. Since $\gamma_{n}\left(C_{2 n}\right)=B_{2 n-2}$, 
it follows from Proposition 3.6 (5) that $H_{\phi}\left(B_{2 n} \mid C_{2 n}\right)=H_{\phi}\left(B_{2 n} \mid B_{2 n-2}\right)$ for all $n \geq 1$. Now let us proceed as in the proof of [36, Corollary 2.4]. Choose $n_{0}$ such that $\operatorname{dim} Z\left(B_{2 n_{0}+2}\right)=\operatorname{dim} Z\left(B_{2 n_{0}}\right)$. Let $\vec{d}$ be the dimension vector of $B_{2 n_{0}}$, $\Lambda$ the inclusion matrix of $B_{2 n_{0}} \subset B_{2 n_{0}+1}$, and $\left(s_{k}\right)$ the trace vector of $B_{2 n_{0}}$ with respect to the trace $\phi$. Then according to [36, Corollary 2.3], $\left(\Lambda \Lambda^{t}\right)^{n} \vec{d}$ is the dimension vector of $B_{2 n_{0}+2 n}$ and $\left(\lambda^{n} s_{k}\right)_{k}$ is the trace vector of $B_{2 n_{0}+2 n}$ with respect to $\phi$ for any $n \geq 0$ where $\lambda=[M: N]_{0}^{-1}$. Hence by [33, Theorem 6.2] (also [35]), we have for every $n \geq 1$

$$
H_{\phi}\left(B_{2 n_{0}+2 n} \mid B_{2 n_{0}+2 n-2}\right)=\sum_{k, l}\left(\Lambda \Lambda^{t}\right)_{k l}\left(\left(\Lambda \Lambda^{t}\right)^{n-1} \vec{d}\right)_{k}\left(\lambda^{n} s_{l}\right) \log \frac{\left(\left(\Lambda \Lambda^{t}\right)^{n} \vec{d}\right)_{l}\left(\lambda^{n-1} s_{k}\right)}{\left(\left(\Lambda \Lambda^{t}\right)^{n-1} \vec{d}\right)_{k}\left(\lambda^{n} s_{l}\right)}
$$

Since $\left(s_{k}\right)$ is the Perron-Frobenius eigenvector of $\Lambda \Lambda^{t}$ with the eigenvalue $\lambda$, we have

$$
\lim _{n \rightarrow \infty} \frac{\left(\left(\Lambda \Lambda^{t}\right)^{n} \vec{d}\right)_{l}\left(\lambda^{n-1} s_{k}\right)}{\left(\left(\Lambda \Lambda^{t}\right)^{n-1} \vec{d}\right)_{k}\left(\lambda^{n} s_{l}\right)}=\lambda^{-2}
$$

for all $k, l$. Therefore

$$
H_{\phi}(A \mid \Gamma(A))=\lim _{n \rightarrow \infty} H_{\phi}\left(B_{2 n_{0}+2 n} \mid B_{2 n_{0}+2 n-2}\right)=\log \lambda^{-2},
$$

as desired.

Following [33], we define the number $\lambda_{\phi}(A, \Gamma(A))$ by

$$
\lambda_{\phi}(A, \Gamma(A))=\max \left\{\lambda \geq 0: E_{\Gamma(A)}(x) \geq \lambda x, x \in A_{+}\right\},
$$

where $E_{\Gamma(A)}$ is the conditional expectation $A \rightarrow \Gamma(A)$ with respect to $\phi$. In view of Theorem 1.1, we can consider $\lambda_{\phi}(A, \Gamma(A))^{-1}$ as a generalized index of $E_{\Gamma(A)}$ when $A$ is not necessarily a factor.

Proposition 5.4. $\lambda_{\phi}(A, \Gamma(A))^{-1} \leq(\operatorname{Index} E)^{2}$.

Proof. Let $\lambda_{\phi}\left(B_{n}, C_{n}\right)$ be defined for the conditional expectation $B_{n} \rightarrow C_{n}$ with respect to $\phi_{n}$. Then as [33, Proposition 2.6], we have $\lambda_{\phi}(A, \Gamma(A))$ $=\lim _{n \rightarrow \infty} \lambda_{\phi}\left(B_{n}, C_{n}\right)$ decreasingly by Proposition 2.4. For each fixed $n \geq 1$, let us use the notations in the proof of Theorem 5.3 (1). Since $\left[D F: D F_{0}\right]_{t}$ $=\left(h_{1} h_{2}\right)^{i t}, F=\left(h_{1} h_{2}\right)^{1 / 2} F_{0}\left(h_{1} h_{2}\right)^{1 / 2}$ follows from [8, Proposition 4.11]. Define $E^{\prime}=\left(h_{1} h_{2}\right)^{1 / 2}\left(E_{0,2}^{\prime} \circ E_{0,1}^{\prime}\right)\left(h_{1} h_{2}\right)^{1 / 2}$. Then $E^{\prime} \in \mathscr{E}\left(M^{\prime}, M_{2}^{\prime}\right)$ because for $x \in M_{2}^{\prime}$

$$
E^{\prime}(x)=E_{0,1}^{\prime}\left(h_{1}\right) E_{0,2}^{\prime}\left(h_{2}\right) x=E_{0,1}\left(h_{1}\right) E_{0,2}\left(h_{2}\right) x=x .
$$


Moreover it follows (see [19]) that

$$
\text { Index } E^{\prime}=\left(E_{0,2}^{\prime} \circ E_{0,1}^{\prime}\right)^{-1}\left(\left(h_{1} h_{2}\right)^{-1}\right)=\lambda^{-2} E_{0,1}\left(h_{1}^{-1}\right) E_{0,2}\left(h_{2}^{-1}\right)=(\operatorname{Index} E)^{2} \text {. }
$$

Since $F_{0}=E_{0,2}^{\prime} \circ E_{0,1}^{\prime} \mid B_{n}$, we have by Theorem 1.1

$$
F(x)=E^{\prime}(x) \geq(\operatorname{Index} E)^{-2} x, \quad x \in\left(B_{n}\right)_{+},
$$

so that $\lambda_{\phi}\left(B_{n}, C_{n}\right) \geq(\operatorname{Index} E)^{-2}$, implying the desired inequality.

The next theorem is an extended version of [7, Theorem 14].

Theorem 5.5. Suppose $E=E_{0}$. Then:

(1) $H_{\phi}(A \mid \Gamma(A)) \leq 2 h_{\phi}(\Gamma) \leq \log \lambda_{\phi}(A, \Gamma(A))^{-1}=2 K_{E}(M \mid N)=2 \log [M: N]_{0}$.

(2) If $\lim _{n \rightarrow \infty} \frac{1}{n} \log k_{n}=0$ (this is the case if $\sup _{n} \frac{1}{n} k_{n}<\infty$ ) where $k_{n}$ is the number of simple summands of $B_{n}$, then $H_{\phi}(A \mid \Gamma(A))=2 h_{\phi}(\Gamma)$.

(3) If $N \subset M$ has finite depth (in particular, if Index $E<4$ ), then the numbers in (1) are all identical together with $\log [A: \Gamma(A)]$.

Proof. (1) By Lemma 4.1, $\Gamma$ is a 2 -shift on the tower $\left\{B_{n}\right\}$. The results [36, Proposition 2.1 and Corollary 2.2] hold for $\left\{B_{n}\right\}$ and $\lambda$ $=[M: N]_{0}^{-1}$. Furthermore we have by Theorems $1.3,1.4$ and 2.6

$$
H_{\phi}\left(B_{2 n}\right) \leq \frac{1}{2} K_{E_{1} \ldots \ldots E_{2 n}}\left(M_{2 n} \mid M\right)=\frac{1}{2} \log \left[M_{2 n}, M\right]_{0}=-n \log \lambda .
$$

Thus we conclude that $\left\{B_{n}\right\}$ is a locally standard tower for $\lambda^{2}$ with period 4 in the sense of [7, Definition 3]. Hence [7, Theorem 8] implies that

$$
H_{\phi}(A \mid \Gamma(A)) \leq 2 h_{\phi}(\Gamma) \leq 2 \log [M: N]_{0} \leq \log \lambda_{\phi}(A, \Gamma(A))^{-1} .
$$

Since $\lambda_{\phi}(A, \Gamma(A))^{-1} \leq[M: N]_{0}^{2}$ by Proposition 5.4, we obtain the conclusion.

(2) For $n \geq 0$, let $K_{n}$ be the set of simple summands of $B_{2 n}$. Then by assumption, $\lim _{n \rightarrow \infty} \frac{1}{n} \log \left|K_{n}\right|=0$ where $|\cdot|$ denotes the cardinal number. We denote by $\left(d_{k}^{(n)}\right)_{k \in K_{n}}$ the dimension vector of $B_{2 n}$ and by $\left(t_{k}^{(n)}\right)_{k \in K_{n}}$ the trace vector of $B_{2 n}$ with respect to the trace $\phi$. Moreover let $\left(a_{k l}^{(n)}\right)_{k \in K_{n}, l \in K_{n+1}}$ be the inclusion matrix of $B_{2 n} \subset B_{2 n+2}$ and let $L_{n}=\left\{(k, l) \in K_{n} \times K_{n+1}: a_{k l}^{(n)}>d_{k}^{(n)}\right\}$. To simplify the notation, we define as in $[6,7]$

$$
I_{\phi}\left(B_{2 n}\right)=\sum_{k \in K_{n}} d_{k}^{(n)} t_{k}^{(n)} \log \frac{d_{k}^{(n)}}{t_{k}^{(n)}}
$$

and analogously $I_{\phi}\left(C_{2 n}\right)$. For each $n \geq 1$, since the mirroring $\gamma_{n}$ maps $B_{2 n-2}$ $\subset B_{2 n}$ to $C_{2 n} \subset B_{2 n}$, the inclusion matrix of $C_{2 n} \subset B_{2 n}$ coincides with $\left(a_{k l}^{(n-1)}\right)_{k \in K_{n-1}, l \in K_{n}}$ and the dimension vector of $C_{2 n}$ coincides with $\left(d_{k}^{(n-1)}\right)_{k \in K_{n-1}}$ 
under the identification of respective simple summands via $\gamma_{n}$. Also let $\left(\tilde{t}_{k}^{(n)}\right)_{k \in K_{n}}$ be the trace vector of $B_{2 n}$ corresponding to $C_{2 n} \subset B_{2 n}$, which is a permutation of $\left(t_{k}^{(n)}\right)$ via $\gamma_{n}$. Then according to [33, Theorem 6.2], we have

$$
H_{\phi}\left(B_{2 n} \mid C_{2 n}\right)=I_{\phi}\left(B_{2 n}\right)-I_{\phi}\left(B_{2 n-2}\right)+\sum_{(k, l) \in L_{n-1}} d_{k}^{(n-1)} a_{k l}^{(n-1)} \tilde{t}_{l}^{(n)} \log \frac{d_{k}^{(n-1)}}{a_{k l}^{(n-1)}},
$$

because $I_{\phi}\left(C_{2 n}\right)=I_{\phi}\left(B_{2 n-2}\right)$ by Proposition 3.6. Now let $\tilde{\lambda}=\max \{\lambda, 1-\lambda\}$. Then $0<\tilde{\lambda}<1$ except the trivial case $N=M$. Since $B_{2 n}$ contains mutually commuting projections $e_{1}, e_{3}, \ldots, e_{2 n-1}$, and since

$$
\phi\left(f_{1} f_{3} \cdots f_{2 n-1}\right)=\phi\left(f_{1}\right) \phi\left(f_{3}\right) \cdots \phi\left(f_{2 n-1}\right) \leq \tilde{\lambda}^{n}
$$

for $f_{2 i-1}=e_{2 i-1}$ or $f_{2 i-1}=1-e_{2 i-1}, \quad 1 \leq i \leq n$, we get $t_{k}^{(n)} \leq \tilde{\lambda}^{n}$ for all $n, k$. Furthermore according to [36, Corollary 2.2], we get $a_{k l}^{(n)} \leq[M: N]_{0}$ for all $n, k, l$. These imply that

$$
\begin{aligned}
0 & \leq-\sum_{(k, l) \in L_{n-1}} d_{k}^{(n-1)} a_{k l}^{(n-1)} \tilde{t}_{l}^{(n)} \log \frac{d_{k}^{(n-1)}}{a_{k l}^{(n-1)}} \\
& \leq \sum_{(k, l) \in L_{n-1}}\left(a_{k l}^{(n-1)}\right)^{2} \tilde{t}_{l}^{(n)} \log a_{k l}^{(n-1)} \\
& \leq\left|K_{n-1}\right|\left|K_{n}\right| \tilde{\lambda}^{n}[M: N]_{0}^{2} \log [M: N]_{0},
\end{aligned}
$$

which tends to 0 as $n \rightarrow \infty$. On the other hand, it follows (see [6, Proposition 16], [7, Proposition 4]) that

$$
0 \leq 2 H_{\phi}\left(B_{2 n}\right)-I_{\phi}\left(B_{2 n}\right) \leq \log \left|K_{n}\right| \text {. }
$$

Therefore by Propositions 5.1 and 4.2

$$
H_{\phi}(A \mid \Gamma(A))=\lim _{n \rightarrow \infty} \frac{1}{n} \sum_{j=1}^{n} H_{\phi}\left(B_{2 j} \mid C_{2 j}\right)=\lim _{n \rightarrow \infty} \frac{1}{n} I_{\phi}\left(B_{2 n}\right)=2 h_{\phi}(\Gamma) .
$$

(3) As [36, Corollary 6.7] (also [14, 4.6.6]), we see that if Index $E<4$, then $N \subset M$ has finite depth. Thus the desired assertion is immediate from (1) and Theorem 5.3 (3) together with Proposition 3.7 (4).

Finally in the type $\mathrm{II}_{1}$ case, we have the next proposition (without the assumption $E_{N}=E_{0}$ ) in view of the proof of Theorem 5.5 (2).

Proposition 5.6. Let $N \subset M$ be type $\mathrm{II}_{1}$ factors and $H(\Gamma)$ the entropy of the canonical shift $\Gamma$ for $N \subset M$. Then:

(1) $H(A \mid \Gamma(A)) \leq 2 H(\Gamma)$.

(2) If $\lim _{n \rightarrow \infty} \frac{1}{n} \log k_{n}=0$ where $k_{n}$ is the number of simple summands of $B_{n}$, then $H(A \mid \Gamma(A))=2 H(\Gamma)$. 


\section{§6. Examples}

In this section, we present two simple examples to illustrate the results in $\S \S 4$ and 5 . In the following, we use the same notations as before.

Example 6.1. Let $M=R$ be the hyperfinite type $\mathrm{II}_{1}$ factor, $N=R_{\lambda}$ Jones' subfactor [22] of $M$ with $[M: N]=\lambda^{-1}$, and $\Gamma$ the canonical shift for $N$ $\subset M$. It follows from Proposition 3.2 that $\gamma_{n}\left(e_{k}\right)=e_{2 n-k}$ for every $n \geq 1$ and $1 \leq k \leq 2 n-1$. Hence

$$
\Gamma\left(e_{n}\right)=\gamma_{n+1}\left(\gamma_{n}\left(e_{n}\right)\right)=\gamma_{n+1}\left(e_{n}\right)=e_{n+2}, \quad n \geq 1 .
$$

Suppose $\lambda \geq 1 / 4$. Then it is known (see $[14,4.7 . \mathrm{b}]$ ) that $M^{\prime} \cap M_{n}$ $=\left\langle 1, e_{1}, \ldots, e_{n-1}\right\rangle$. Hence $A=\left\{e_{n}: n \geq 1\right\}^{\prime \prime}(\simeq R)$, so that $\Gamma$ coincides with $\sigma_{\lambda}^{2}$ where $\sigma_{\lambda}$ is a special case of the shifts discussed in $[5,6]$. We have by $[6$, Example 2]

$$
\frac{1}{2} H(A \mid \Gamma(A))=H(\Gamma)=2 H\left(\sigma_{\lambda}\right)=H(M \mid N)=\log \lambda^{-1} .
$$

Next suppose $\lambda<1 / 4$ and $t(1-t)=\lambda, t>0$. We get

$$
\begin{aligned}
H(\Gamma) & =\lim _{n \rightarrow \infty} \frac{1}{n} H\left(B_{2 n}\right) \geq \lim _{n \rightarrow \infty} \frac{1}{n} H\left(\left\langle 1, e_{1}, \ldots, e_{2 n-1}\right\rangle\right) \\
& =2 H\left(\sigma_{\lambda}\right)=2 \eta(t)+2 \eta(1-t)=H(M \mid N)
\end{aligned}
$$

by [7, Theorem 1], [6, Example 2] and [33, Corollary 5.3]. On the other hand,

$$
H(\Gamma) \leq \frac{1}{2}\left\{H(M \mid N)+H\left(M_{1} \mid M\right)\right\}=H(M \mid N)
$$

by Corollary 4.4 (1) and [21, Proposition 8.4]. Hence $H(\Gamma)=H(M \mid N)$. Moreover since the Bratteli diagram for the tower $B_{0} \subset B_{1} \subset B_{2} \subset \cdots$ is Pascal's triangle (see $[14$, p. 231]), we have $H(A \mid \Gamma(A))=2 H(\Gamma)$ by Proposition 5.6 (2). Therefore $\frac{1}{2} H(A \mid \Gamma(A))=H(\Gamma)=H(M \mid N)$ for any $\lambda$.

Example 6.2. Let us consider $M=N \otimes B \supset N=N \otimes \mathbb{C}$ where $N$ is any factor and $B=M_{m}(C)$. Let $\varphi_{0} \in \mathscr{E}(B)$ and $h=d \varphi_{0} / d \tau$ where $\tau$ is the normalized trace on $B$. Define $E \in \mathscr{E}(M, N)$ by $E=\mathrm{id}_{N} \otimes \varphi_{0}$ and $\varphi_{1} \in \mathscr{E}(B)$ by $d \varphi_{1} / d \tau$ $=h^{-1} / \tau\left(h^{-1}\right)$. Then it follows (see [21, Example 8.3]) that the basic constructions $E_{n} \in \mathscr{E}\left(M_{n}, M_{n-1}\right), n \geq 1$, iterated from $E$ are given as follows:

$$
\begin{aligned}
& M_{n}=M \otimes B^{(n)}=M_{n-1} \otimes B, \\
& E_{2 n-1}=\mathrm{id}_{M_{2 n-2}} \otimes \varphi_{1}, \quad E_{2 n}=\mathrm{id}_{M_{2 n-1}} \otimes \varphi_{0},
\end{aligned}
$$


where $B^{(n)}=\bigotimes_{1}^{n} B$. Moreover it is easy to see that the mirrorings $\gamma_{n}$ on $M^{\prime} \cap M_{2 n}=B^{(2 n)}$ are given by

$$
\gamma_{n}\left(a_{1} \otimes a_{2} \otimes \cdots \otimes a_{2 n-1} \otimes a_{2 n}\right)=a_{2 n}^{t} \otimes a_{2 n-1}^{t} \otimes \cdots \otimes a_{2}^{t} \otimes a_{1}^{t},
$$

where $a^{t}$ denotes the transpose of $a$. Therefore

$$
\begin{aligned}
\Gamma\left(a_{1} \otimes a_{2} \otimes \cdots \otimes a_{2 n-1} \otimes a_{2 n}\right) & =\gamma_{n+1}\left(a_{2 n}^{t} \otimes a_{2 n-1}^{t} \otimes \cdots \otimes a_{2}^{t} \otimes a_{1}^{t} \otimes 1 \otimes 1\right) \\
& =1 \otimes 1 \otimes a_{1} \otimes a_{2} \otimes \cdots \otimes a_{2 n-1} \otimes a_{2 n},
\end{aligned}
$$

so that $\Gamma$ is the unilateral shift on $(A, \phi)=\otimes_{1}^{\infty}\left(B^{(2)}, \varphi_{1} \otimes \varphi_{0}\right)$. This example clarifies that $\phi$ is not generally invariant for $\gamma_{n}$ but $\Gamma$ preserves $\phi$. By [10, Corollaire 10], we have

$$
h_{\phi}(\Gamma)=S\left(\varphi_{1} \otimes \varphi_{0}\right)=S\left(\varphi_{0}\right)+S\left(\varphi_{1}\right)=\frac{1}{2}\left\{K_{E}(M \mid N)+K_{E_{1}}\left(M_{1} \mid M\right)\right\} .
$$

When $\varphi_{0}=\tau$ and hence $\varphi_{1} \otimes \varphi_{0}$ is the trace on $B^{(2)}$, we get by $[6$, Example 1$]$

$$
\frac{1}{2} H_{\phi}(A \mid \Gamma(A))=h_{\phi}(\Gamma)=2 \log m=\log [M: N]_{0} .
$$

Also when $\varphi_{0} \neq \tau$ (hence $A$ is a type III factor), we get (see [21, Theorem 6.6], [20, Proposition 3.6 and Example 4.6])

$$
\frac{1}{2} H_{\phi}(A \mid \Gamma(A)) \leq S\left(\varphi_{0}\right)+S\left(\varphi_{1}\right)=h_{\phi}(\Gamma) .
$$

\section{References}

[1] Araki, H., Relative entropy of states of von Neumann algebras, Publ. RIMS, Kyoto Univ., 11 (1976), 809-833.

[2] - Relative entropy for states of von Neumann algebras II, Publ. RIMS, Kyoto Univ., 13 (1977), 173-192.

[ 3 ] Bailet, M., Denizeau, Y. and Havet, J. F., Indice d'une esperance conditionnelle, Compositio Math., 66 (1988), 199-236.

[4] Bratteli, O. and Robinson, D. W., Operator Algebras and Quantum Statistical Mechanics II, Springer-Verlag, New York-Heidelberg-Berlin, 1981.

[5] Choda, M., Shifts on the hyperfinite $\mathrm{II}_{1}$-factor, J. Operator Theory, 17 (1987), 223-235.

[6] - Entropy for *-endomorphisms and relative entropy for subalgebras, J. Operator Theory, to appear.

[7] - Entropy for canonical shifts, Trans. Amer. Math. Soc., to appear.

[8] Combes, F. and Delaroche, C., Groupe modulaire d'une espérance conditionnelle dans une algèbre de von Neumann, Bull. Soc. Math. France, 103 (1975), 385-426.

[9] Connes, A., On the spatial theory of von Neumann algebras, J. Funct. Anal., 35 (1980), 153-164. 
[10] Connes, A., Entropie de Kolmogoroff-Sinai et mécanique statistique quantique, C. R. Acad. Sci. Paris Sér. I, 301 (1985), 1-6.

[11] Connes, A., Narnhofer, H. and Thirring, W., Dynamical entropy of $C^{*}$ algebras and von Neumann algebras, Commun. Math. Phys., 112 (1987), 691-719.

[12] Connes, A. and St $\phi$ rmer, E., Entropy for automorphisms of $\mathrm{II}_{1}$ von Neumann algebras, Acta Math., 134 (1975), 289-306.

[13] Dixmier, J. and Maréchal, O., Vecteurs totalisateurs d'une algèbre de von Neumann, Commun. Math. Phys., 22 (1971), 44-50.

[14] Goodman, F. M., de la Harpe, P. and Jones, V. F. R., Coxeter Graphs and Towers of Algebras, Springer-Verlag, New York, 1989.

[15] Haagerup, U., Operator valued weights in von Neumann algebras, I, II, J. Funct. Anal., 32 (1979), 175-206; 33 (1979), 339-361.

[16] Hamachi, T. and Kosaki, H., Index and flow of weights of factors of type III, Proc. Japan Acad., 64A (1988), 11-13.

[17] - Orbital factor map, preprint.

[18] Havet, J. F., Esperance conditionnelle minimale, preprint.

[19] Hiai, F., Minimizing indices of conditional expectations onto a subfactor, Publ. RIMS, Kyoto Univ., 24 (1988), 673-678.

[20] - Minimum index for subfactors and entropy, J. Operator Theory, to appear.

[21] — Minimum index for subfactors and entropy. II, J. Math. Soc. Japan, 43 (1991), $347-379$.

[22] Jones, V. F. R., Index for subfactors, Invent. Math., 72 (1983), 1-25.

[23] Kawakami, S., Some remarks on index and entropy for von Neumann subalgebras, Proc. Japan Acad., 65A (1989), 323-325.

[24] Kosaki, H., Extension of Jones' theory on index to arbitrary factors, J. Funct. Anal., 66 (1986), 123-140.

[25] — Characterization of crossed product (properly infinite case), Pacific J. Math., 137 (1989), 159-167.

[26] Kosaki, H. and Longo, R., A remark on the minimal index of subfactors, preprint.

[27] Loi, P. H., On the theory of index and type III factors, C. R. Acad. Sci. Paris Sér. I, 305 (1987), 423-426.

[28] Longo, R., Solution of the factorial Stone-Weierstrass conjecture. An application of the theory of standard split $W^{*}$-inclusions, Invent. Math., 76 (1984), 145-155.

[29] - , Simple injective subfactors, Adv. in Math., 63 (1987), 152-171.

[30] - Index of subfactors and statistics of quantum fields. I, Commun. Math. Phys., 126 (1989), 217-247.

[31] Ocneanu, A., Quantized groups, string algebras and Galois theory for algebras, preprint.

[32] Petz, D., Properties of the relative entropy of states of von Neumann algebras, Acta Math. Hungar., 47 (1986), 65-72.

[33] Pimsner, M. and Popa, S., Entropy and index for subfactors, Ann. Sci. École Norm. Sup. Sér. 4, 19 (1986), 57-106.

[34] — - Iterating the basic construction, Trans. Amer. Math. Soc., 310 (1988), 127-133.

[35] - Finite dimensional approximation of pairs of algebras and obstractions for the index, J. Funct. Anal., 98 (1991), 270-291.

[36] Popa, S., Classification of subfactors: the reduction to commuting squares, Invent. Math., 101 (1990), 19-43.

[37] Strătilă, Ş., Modular Theory in Operator Algebras, Editura Academiei and Abacus Press, Tunbridge Wells, 1981.

[38] Takesaki, M., Conditional expectations in von Neumann algebras, J. Funct. Anal., 9 (1972), 
306-321.

[39] Umegaki, H., Conditional expectation in an operator algebra, Tôhoku Math. J., 6 (1954), 177-181.

[40] - Conditional expectation in an operator algebra, IV (entropy and information), Kōdai Math. Sem. Rep., 14 (1962), 59-85.

[41] Watatani, Y., Index for $C^{*}$-subalgebras, Memoirs Amer. Math. Soc., no. 424, 1990. 
